\title{
Abundance analysis of turn-off and early subgiant stars in the globular cluster 47 Tuc (NGC 104) ${ }^{\star}$
}

\author{
E. Carretta ${ }^{1}$, R. G. Gratton ${ }^{1}$, A. Bragaglia ${ }^{2}$, P. Bonifacio ${ }^{3}$, and L. Pasquini ${ }^{4}$ \\ 1 INAF - Osservatorio Astronomico di Padova, Vicolo dell'Osservatorio 5, 35122 Padova, Italy \\ 2 INAF - Osservatorio Astronomico di Bologna, Via Ranzani 1, 40127 Bologna, Italy \\ ${ }^{3}$ INAF - Osservatorio Astronomico di Trieste, via Tiepolo 11, 34131 Trieste, Italy \\ ${ }^{4}$ ESO - European Southern Observatory
}

Received 22 September 2003 / Accepted 5 November 2003

\begin{abstract}
We used the UVES spectrograph on Kueyen (VLT UT2) to perform abundance analysis of stars at the base of the giant branch (RGB) and at the main sequence turn-off in the globular cluster 47 Tuc (NGC 104). High dispersion spectra $(R \gtrsim 40000)$ for 3 dwarfs and 9 subgiants were analyzed. We found an overall $[\mathrm{Fe} / \mathrm{H}]$ value of $-0.67 \pm 0.01 \pm 0.04, \mathrm{rms}=$ 0.05 dex. The mean value obtained from stars at the base of the RGB is virtually the same as that obtained from the dwarfs. $\mathrm{Na}$ and $\mathrm{O}$ abundances show a star-to-star scatter and are anti-correlated, not unlike previous findings in more metal-poor clusters. The extension of the anticorrelation is not as extreme as found in other clusters, but it is clearly present in unevolved stars. Al abundances do not show a significant spread; this could be indicative that p-captures in high temperature regions are less likely to occur in metal-rich clusters. The $[\alpha / \mathrm{Fe}]$ ratios suggest a slight excess of Ti with respect to field stars of similar metallicities. We found an enhanced odd-even effect for Fe-group elements. In particular, the $[\mathrm{Mn} / \mathrm{Fe}] \mathrm{ratio}$ is almost 0.2 dex deficient with respect to the mean value of the dissipative component of field stars in the solar neighborhood. While the theoretical and observational framework for Mn is still poorly understood, the chemistry of 47 Tuc, coupled with its kinematics and its somewhat younger age, might give hints favouring an origin in a formely independent, possibly larger, subsystem of our Galaxy.
\end{abstract}

Key words. stars: abundances - stars: atmospheres - stars: evolution - stars: population II - Galaxy: globular clusters: general - Galaxy: globular clusters: individual: 47 Tuc

\section{Introduction}

The galactic globular clusters (GCs) are important for a large set of astrophysical problems, ranging from stellar evolution to cosmology. Their chemical compositon is a fundamental probe of the early chemical evolution in the galactic environment. However, before using globular clusters as tracers of the early chemical conditions in the Galaxy, one has to disentangle the original compositions from any effect which could have resulted in a pollution of their photospheres. Possible effects include (i) possible self-enrichment of the protoclouds from which the GCs originate (Cayrel 1986; Brown et al. 1991, 1995); (ii) changes in abundances derived from the internal evolution of the stars (both canonical or non-canonical mixing, see Kraft 1994; Gratton et al. 2000b); and (iii) modifications of the composition due to a possible second generation of stars born from the low velocity wind of intermediate mass

Send offprint requests to: E. Carretta,

e-mail: carretta@pd.astro.it

* Based on data collected at the European Southern Observatory, Chile, in the course of the ESO-Large program 165.L-0263. globular cluster stars (Parmentier et al. 1999; Parmentier \& Gilmore 2001).

A growing body of evidence shows that the usual assumption of globular cluster stars being exactly coeval and chemically homogeneous (the basis of their classification as a pure simple stellar population) is unreliable. Useful records of the first phases of formation and early evolution of GCs are hidden in the photospheric abundances of stars presently observed. Once correctly interpreted, they may provide an understanding of the origin and evolution of GCs which may not be achieved by other means.

The most noticeable of these anomalies are those involving the light element $\mathrm{C}, \mathrm{N}, \mathrm{O}$ (for exhaustive reviews see e.g. Smith 1987; Smith \& Mateo 1990; Kraft 1994 and references therein), as detected from molecular abundances of $\mathrm{CN}, \mathrm{CH}$, $\mathrm{NH}$ and $\mathrm{OH}$, often accompanied by correlated anomalies in atomic species such as $\mathrm{O}, \mathrm{Na}, \mathrm{Al}$ and $\mathrm{Mg}$ (see the survey by the Lick-Texas group).

The observed abundance pattern may be explained by H-burning at high temperatures, where reactions and products of the $\mathrm{CNO}$, $\mathrm{NeNa}$ and $\mathrm{MgAl}$ cycles occur. 
Denisenkov \& Denisenkova (1990), Langer et al. (1993) have shown that production of ${ }^{23} \mathrm{Na}$ and ${ }^{27} \mathrm{Al}$ from proton captures on ${ }^{22} \mathrm{Ne}$ and ${ }^{25} \mathrm{Mg}$ is possible even in low mass stars, in regions close to the ON-burning shell.

To explain the complex observational picture, there are presently two main lines of thought. The first (see Cottrell \& Da Costa 1981; Brown \& Wallerstein 1992) considers primordial inhomogeneities, i.e. already existing in the gas from which the cluster stars formed, or released from the very early generation of massive stars born and evolved in GCs. This scenario is based on the belief that $\mathrm{Na}$ and $\mathrm{Al}$ cannot be synthetized in the low mass stars presently observed in GCs, so that the overabundances of these elements, and the correlated $\mathrm{CN}$-enhancements, must be due to the yields of more massive stars that polluted the next generation of cluster stars.

The second scenario explains the $\mathrm{CN}$ and anticorrelated anomalies as due to internal deep mixing, that brings up to the surface fresh ${ }^{14} \mathrm{~N}$ from $\mathrm{CN}$ processes, depleting at the same time the abundance of ${ }^{12} \mathrm{C}$ of the surface layers. The constant decrease of the isotopic ratio ${ }^{12} \mathrm{C} /{ }^{13} \mathrm{C}$ up to values near the equilibrium ratio of 3.5 (see the review by Suntzeff 1993) supports this mechanism. All stars experience a mixing phase (the standard first dredge-up) during the first ascent on the giant branch; however, to explain the fact that only some of the stars are $\mathrm{CN}$-strong, one has to assume the existence of a mechanism of extra mixing.

Following the model of Sweigart \& Mengel (1979), this additional mechanism could be represented by meridional circulation currents driven by core rotation. To explain the observations, these currents should be able to reach the region of the ON process, bringing up to the surface processed $\mathrm{N}$-rich and O-poor material that contributes to the $\mathrm{CN}$ enhancements even in the case of large $\mathrm{C}$ depletions. However, in the model of Sweigart \& Mengel (1979) a gradient of mean molecular weight does inhibit the formation of circulation currents (Tassoul \& Tassoul 1984), and so the additional mixing, below a critical level $\left(\log L / L_{\odot} \sim 2.2\right)$ on the RGB. This is the point of maximum penetration of the convective envelope, succesively crossed by the H-burning shell propagating outward (the so-called RGB-bump).

Observations show the opposite: anomalous $\mathrm{CN}$ abundances exist at considerably lower luminosities than predicted. Furthermore, a radial dependence in the distributions of $\mathrm{CN}$-strong and $\mathrm{CN}$-weak stars (the first being more centrally concentrated) seems to be present in 47 Tuc and possibly in other clusters (Norris 1987), and this is not easily explained by the mixing scenario.

Recently we definitively settled this issue by finding a clear Na-O anticorrelation among unevolved (turn-off) stars in the globular cluster NGC 6752 (Gratton et al. 2001, hereafter Paper I). These stars do not meet the constraints of high central temperature and extended envelope required to self-produce and bring to the surface the synthetized proton-capture elements. The same seems to hold also in the more metal-poor cluster NGC 6397 (Carretta et al. 2003a, in preparation).

The Na-O anticorrelation is apparently limited to the dense environments of GCs: there are almost no stars showing CN-strong or Na-rich composition in the field, where the chances for pollution are much less than in the denser GCs. Gratton et al. (2000b) convincingly showed that the abundances of elements heavier than $\mathrm{CN}$ (e.g. $\mathrm{Na}$ and $\mathrm{O}$ ) are unaltered in field stars of whatever evolutionary stages, from pre-main sequence up to the horizontal branch (HB) phase.

There are several reasons that make 47 Tuc a preferred target for studying the complex issue of chemical anomalies in globular cluster stars:

- among the few most nearby clusters, it has reasonably low interstellar extinction;

- 47 Tuc is traditionally adopted as the classical template of the high-metallicity tail of the metal distribution of globular clusters;

- as a typical metal-rich cluster, 47 Tuc is considered a pillar of the globular cluster distance scale based on the main sequence fitting method (see e.g. Carretta et al. 2000; Gratton et al. 2003a);

- this cluster is very well studied, from photometric point of view, and large databases in different photometric systems exist. However, despite its proximity, it has not been extensively studied with high-resolution spectroscopy. Instead, a sparse number of its giant stars have been observed, mostly for calibrating purposes, as in the Norris \& Da Costa (1995) study of $\omega$ Cen;

- finally, the relevance of studying 47 Tuc is evident, since most mixing mechanisms are less and less efficient as the global metal abundance increases, due to the strengthening of the molecular weight barrier (Sweigart \& Mengel 1979). Hence, in metal-rich environments it should be easier to investigate the primordial component of the so-called chemical anomalies in globular cluster stars.

Several studies at medium and low resolution have been performed in the past. They were aimed to extract information from $\mathrm{CN}$ and $\mathrm{CH}$ band strengths in main sequence and turn-off stars of 47 Tuc (Hesser 1978; Hesser \& Bell 1980; Bell et al. 1983; Briley et al. 1991, 1996; Cannon et al. 1998 and references therein). Pushing to the limit the size and limit magnitude of the samples, these investigations also uncovered many features of the $\mathrm{CH}$ and $\mathrm{CN}$ distributions among unevolved dwarfs in this cluster.

The favourable location of the ESO VLT complex in the southern hemisphere, coupled with the high efficiency of the UVES spectrograph, now allow us to obtain high-resolution spectra at a $S / N$ adequate for a precise, detailed abundance analysis of several elements in 47 Tuc dwarfs.

\section{Observations}

Observations were carried out in three different runs (September 2000; August and October 2001) with the spectrograph UVES at the second 8m Unit Telescope of the VLT (Paranal ESO Observatory); each run consisted of 6 nights assigned to the ESO Large Program 165.L-0263. Due to poor weather conditions in the September 2000 run, we were not able to complete the acquisition of the spectra of 47 Tuc. Bad weather/seeing also plagued the August 2001 and partially the 


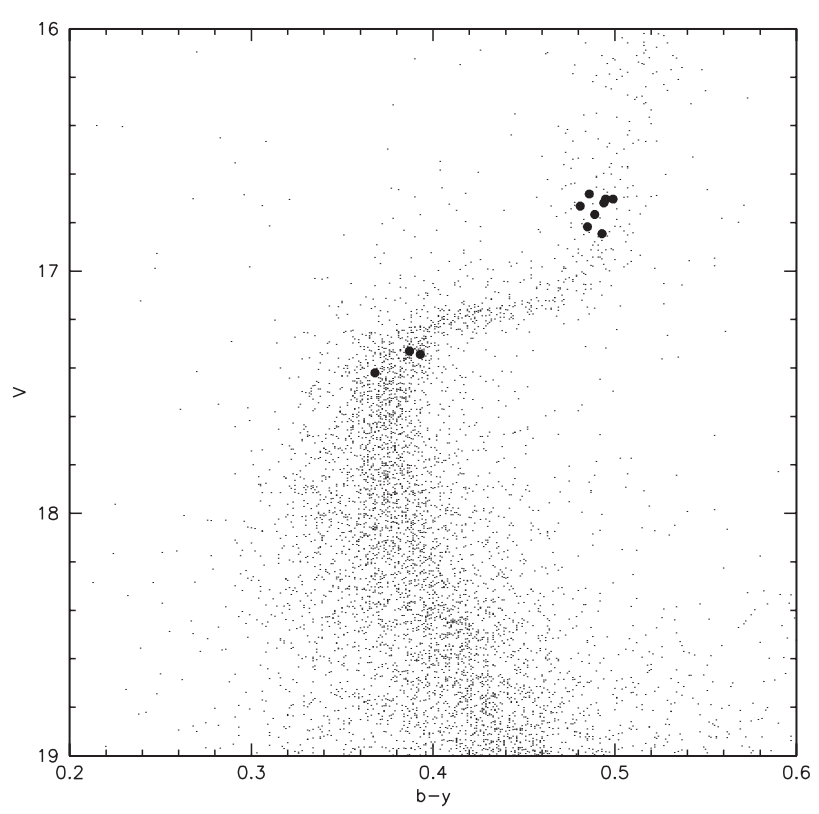

Fig. 1. Positions of program stars (large symbols) on the Strömgren colour-magnitude diagram of 47 Tuc. Photometry is from Grundahl et al. (1999).

October 2001 runs. In total, 5 candidate turn-off stars and 9 candidate subgiant stars were observed. However, one of the turnoff stars turned out to be non-member, both from radial velocity (see Lucatello \& Gratton 2003) and elemental abundances, and for another we were able to gather only a low- $S / N(\sim 15)$ spectrum, useless apart from the membership determination. Hence we have useful observational material (high-resolution, high $S / N$ spectra) only for 9 stars at the base of the RGB and 3 stars at the main sequence turn-off in 47 Tuc.

Following Paper I, we tried to observe stars likely having both strong and weak $\mathrm{CN}$ bands, selecting objects on the basis of Strömgren photometry, using the index $c_{1}$ (increasing with the strength of CN bands, Grundahl et al. 1999).

Selected stars are indicated in Fig. 1, and their relevant data are listed in Table 1.

As for NGC 6397 and NGC 6752 (see Paper I), data were acquired using the dichroic beamsplitter \#2 at UVES. The CD2, centered at $420 \mathrm{~nm}$ (with spectral coverage $\lambda \lambda 356-484 \mathrm{~nm}$ ), was used in the blue arm of the spectrograph; and the CD4 centered at $750 \mathrm{~nm}$ (covering $\lambda \lambda$ 555-946 nm) was adopted in the red arm. Slit length was always 8 arcsec; the slit width was mostly set at 1 arcsec (corresponding to a resolution of 43000 at the order centers). However, this value was slightly modified downward or upward in a few cases, according to the seeing conditions.

Typical exposure times were $2 \mathrm{~h}$ for each subgiant and about $4 \mathrm{~h}$ for each turn-off star, split in multiple exposures. Values of the $S / N$ per pixel (about 5 per resolution element) measured at about $6700 \AA$ are listed in Table 1.

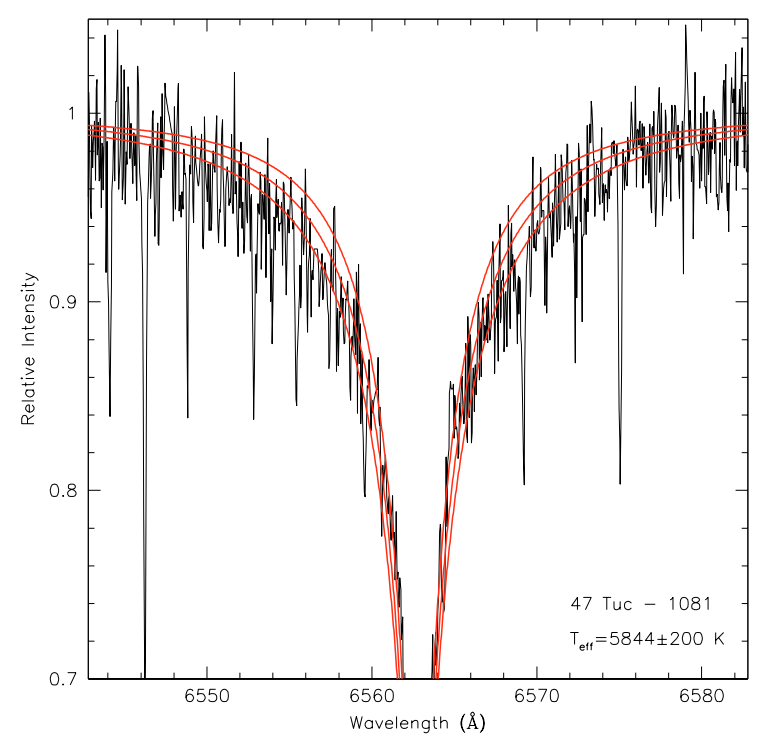

Fig. 2. Derivation of temperature from the $\mathrm{H} \alpha$ profile for star 1081 in 47 Tuc. Thin line: observed profile; thick lines: expected profiles computed for $T_{\text {eff }}=5644,5844$, and $6044 \mathrm{~K}$. In the fitting we used only the regions where the relative intensity is $>0.9$.

\section{Atmospheric parameters and iron abundances}

\subsection{Atmospheric parameters}

Following the procedure adopted in Paper I, we compared effective temperatures from observed colours (both Johnson $B-V$ and Strömgren $b-y$ were used) with spectroscopic temperatures derived from comparison of Balmer line profiles (namely $\mathrm{H} \alpha$ ) with those obtained from spectral synthesis. An example of the fit is shown in Fig. 2 for star 1081.

Since (i) these last temperatures are reddening-free and (ii) they are derived following a uniform procedure for field stars (see Paper I), this approach allows us to compare directly our derived abundances for 47 Tuc with those obtained for field stars. This is a key requisite for accurate distances from the Main Sequence Fitting Method (see Gratton et al. 2003a) ${ }^{1}$.

However, some differences do exist with respect to the analysis performed for stars in NGC 6397 and NGC 6752.

- we adopted individual $T_{\text {eff }} \mathrm{s}$ for subgiant stars, but a unique value (the average of individual ones) for the 3 stars at the main-sequence turn-off. This procedure provides the best agreement with temperatures from line excitation;

- we used new enhancement factors for collisional damping. These are described in detail in Gratton et al. (2003b);

- a new task was designed to iteratively clean the set of lines, disregarding outliers that yield abundances differing more than $2.5 \sigma$ from the average abundance of the remaining lines.

Values of the surface gravity were derived (as in Paper I) from the location of stars in the colour magnitude diagram, when an age of $14 \mathrm{Gyr}$ and corresponding masses were assumed.

1 The adopted temperature scale for field subdwarfs is very close to that of Alonso et al. (1996). 
Table 1. Data for observed stars in 47 Tuc (identifications from Grundahl et al. 1999).

\begin{tabular}{|c|c|c|c|c|c|c|c|c|c|c|c|}
\hline Star & $S / N$ & $\begin{array}{c}v_{r} \\
\mathrm{~km} \mathrm{~s}^{-1}\end{array}$ & $\begin{array}{l}\text { observing } \\
\text { run }\end{array}$ & $B$ & $\bar{V}$ & $u$ & $b$ & $v$ & $y$ & RA(2000) & $\overline{\operatorname{Dec}(2000)}$ \\
\hline \multicolumn{12}{|c|}{ Subgiants } \\
\hline 435 & 40 & $-20.2 \pm 0.6$ & Oct. 2001 & 17.540 & 16.739 & 19.022 & 17.213 & 17.960 & 16.732 & $00: 21: 13.65$ & $-72: 03: 32.41$ \\
\hline 456 & 40 & $-16.1 \pm 0.3$ & Oct. 2001 & 17.537 & 16.746 & 19.016 & 17.256 & 17.989 & 16.767 & $00: 21: 30.42$ & $-72: 05: 35.95$ \\
\hline 433 & 40 & $-23.0 \pm 0.2$ & Oct. 2001 & 17.502 & 16.723 & 18.993 & 17.198 & 17.945 & 16.703 & $00: 21: 49.24$ & $-72: 02: 54.06$ \\
\hline 478 & 30 & $-20.4 \pm 0.3$ & Oct. 2001 & 17.609 & 16.827 & 19.131 & 17.339 & 18.076 & 16.846 & $00: 21: 08.17$ & $-71: 58: 47.54$ \\
\hline 201600 & 40 & $-9.5 \pm 0.4$ & Oct. 2001 & 17.470 & 16.669 & 19.001 & 17.168 & 17.920 & 16.682 & $00: 21: 52.55$ & $-72: 05: 27.66$ \\
\hline 429 & 35 & $-19.3 \pm 0.2$ & Sep. 2001 & 17.499 & 16.699 & 19.022 & 17.202 & 17.953 & 16.703 & 00:20:18.60 & $-72: 01: 11.40$ \\
\hline 201075 & 40 & $-10.2 \pm 0.2$ & Sep. 2001 & 17.609 & 16.817 & 18.987 & 17.302 & 18.013 & 16.817 & 00:22:00.40 & $-72: 05: 59.40$ \\
\hline 206415 & 40 & $-21.8 \pm 0.3$ & Sep. 2001 & & & 18.940 & 17.213 & 17.932 & 16.719 & $00: 19: 36.40$ & $-71: 58: 57.00$ \\
\hline 482 & 45 & $-0.6 \pm 0.3$ & Aug. 2001 & 17.648 & 16.816 & 19.156 & 17.352 & 18.097 & 16.842 & $00: 21: 10.88$ & $-72: 04: 15.16$ \\
\hline \multicolumn{12}{|c|}{ Dwarfs } \\
\hline 1012 & 30 & $-22.8 \pm 0.2$ & Oct. 2001 & 17.942 & 17.361 & 19.053 & 17.737 & 18.251 & 17.344 & $00: 21: 26.27$ & $-72: 00: 38.73$ \\
\hline 1081 & 45 & $-16.1 \pm 0.2$ & Aug. 2001 & 17.962 & 17.375 & 19.099 & 17.788 & 18.282 & 17.420 & $00: 21: 03.82$ & $-72: 06: 57.74$ \\
\hline 975 & 40 & $-20.2 \pm 0.2$ & Sep. 2000 & 17.919 & 17.322 & 19.068 & 17.717 & 18.247 & 17.330 & $00: 20: 52.30$ & $-71: 58: 01.80$ \\
\hline
\end{tabular}

Values of $S / N$ are estimated at $6700 \AA$; heliocentric radial velocities are from Lucatello \& Gratton (2003).

Estimates of the microturbulent velocity $v_{\mathrm{t}}$ for each star were derived, as usual, by eliminating the trend of abundances with expected line strengths; for this, we used abundances of $\mathrm{Fe} \mathrm{I}$, for which a large number of lines were measured in each star. Unlike Paper I, we found that, for stars at the base of the giant branch, the star-to-star scatter in Fe abundances was reduced if individual values of microturbulent velocity were used.

Finally, the overall model metallicity $[\mathrm{A} / \mathrm{H}]$ was chosen as that of the model atmospheres extracted from the grid of ATLAS models with the overshooting option switched off computed by Castelli ${ }^{2}$, that best reproduce the measured equivalent widths $(E W)$.

Final adopted atmospheric parameters are listed in Table 2.

\subsection{Equivalent widths}

Automatic equivalent width measurements of spectral lines were performed exploiting a recent version of the spectrum analysis package developed in Padova and partially described in Bragaglia et al. (2001) and Carretta et al. (2002). The number of useful lines depends on the metallicity of star and on the $S / N$. The metal abundance being the same for dwarfs and early subgiants in 47 Tuc, we were able to measure similar numbers of Iron lines for the two groups, i.e. from 50 to $60 \mathrm{Fe}$ I lines for the SGB and from 30 to $40 \mathrm{Fe}$ I lines for dwarfs, and about 10 Fe II lines in each group, securing a good database with statistical significance for the following analysis.

From the classical formula by Cayrel (1989) widely used to give an estimate of the error in measured $E W$ as a function of the full width half-maximum, we expect an error of $3.8 \mathrm{~m} \AA$, due to the characteristics of our spectra and their typical $S / N$ ratios (see Table 1). This value can be compared with observations.

An empirical estimate of internal errors in the equivalent widths can be obtained by comparing stars of similar physical

\footnotetext{
2 Available from http://kurucz.harvard.edu/
}
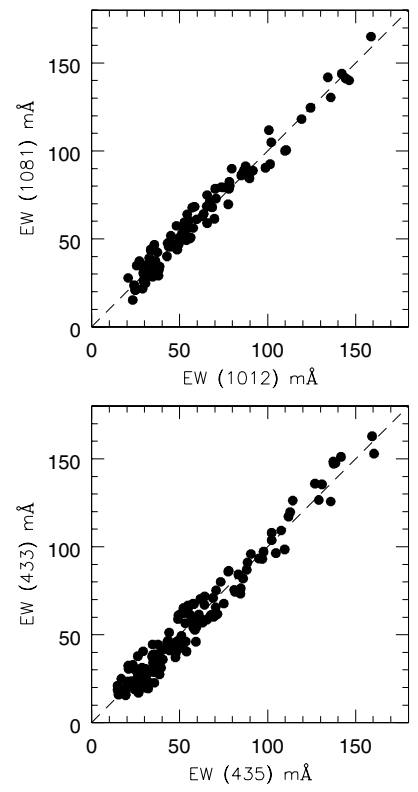

Fig. 3. Comparison between $E W \mathrm{~s}$ measured on the spectra of two dwarfs in 47 Tuc (upper panel; stars 1012 and 1081) and two subgiants (lower panel, stars 433 and 435). The dashed lines indicate equality, and the rms scatters about the average differences amount to $6.8 \mathrm{~m} \AA$ in the case of subgiants and $5.5 \mathrm{~m} \AA$ for TO-stars, in this example.

status and with spectra of similar quality. We performed this exercise by cross-comparing the sets of $E W \mathrm{~s}$ measured for subgiants (discarding from the comparison only the 2 stars with lower $S / N$ ) and turn-off dwarfs separately. From 15 comparisons of subgiants, we derived an average rms scatter of $6.4 \mathrm{~m} \AA$ for the average difference $E W(\operatorname{star} 1)-E W(\operatorname{star} 2)$, based typically on about 140 lines in common between each pair of stars. In the same way, the 3 inter-comparisons of the three dwarfs resulted in an average rms scatter of $7.9 \mathrm{~m} \AA$. If we suppose that errors can be attributed in equal proportions to the stars, we obtain with typical errors in $E W \mathrm{~s}$ of $4.5 \mathrm{~m} \AA$ for subgiants and $5.6 \mathrm{~m} \AA$ for the turn-off stars. Figure 3 shows two of these 
Table 2. Adopted atmospheric parameters and derived Iron abundances for observed stars in 47 Tuc.

\begin{tabular}{|c|c|c|c|c|c|c|c|c|c|c|}
\hline Star & $\begin{array}{l}T_{\text {eff }} \\
(\mathrm{K})\end{array}$ & $\begin{array}{l}\log g \\
(\operatorname{dex})\end{array}$ & $\begin{array}{c}\mathrm{A} / \mathrm{H}] \\
(\mathrm{dex})\end{array}$ & $\begin{array}{c}v_{\mathrm{t}} \\
\left(\mathrm{km} \mathrm{s}^{-1}\right)\end{array}$ & $n$ & {$[\mathrm{Fe} / \mathrm{H}] \mathrm{I}$} & rms & $n$ & {$[\mathrm{Fe} / \mathrm{H}] \mathrm{II}$} & $\mathrm{rms}$ \\
\hline \multicolumn{11}{|c|}{ Subgiants } \\
\hline 435 & 5190 & 3.84 & -0.63 & 0.00 & 55 & -0.64 & 0.17 & 6 & -0.48 & 0.17 \\
\hline 456 & 5142 & 3.84 & -0.68 & 0.50 & 51 & -0.71 & 0.11 & 6 & -0.59 & 0.09 \\
\hline 433 & 5106 & 3.84 & -0.74 & 1.05 & 55 & -0.78 & 0.15 & 6 & -0.57 & 0.11 \\
\hline 478 & 5118 & 3.84 & -0.56 & 0.00 & 59 & -0.59 & 0.20 & 6 & -0.65 & 0.15 \\
\hline 201600 & 5160 & 3.84 & -0.61 & 0.70 & 52 & -0.65 & 0.12 & 9 & -0.63 & 0.13 \\
\hline 429 & 5081 & 3.84 & -0.61 & 1.04 & 67 & -0.65 & 0.16 & 10 & -0.53 & 0.19 \\
\hline 201075 & 5165 & 3.84 & -0.64 & 0.70 & 48 & -0.68 & 0.10 & 10 & -0.59 & 0.14 \\
\hline 206415 & 5112 & 3.84 & -0.67 & 1.05 & 58 & -0.70 & 0.13 & 8 & -0.61 & 0.15 \\
\hline 482 & 5090 & 3.84 & -0.62 & 0.84 & 62 & -0.62 & 0.09 & 9 & -0.36 & 0.14 \\
\hline \multicolumn{11}{|c|}{ Dwarfs } \\
\hline 1012 & 5832 & 4.05 & -0.65 & 1.07 & 38 & -0.64 & 0.21 & 7 & -0.66 & 0.09 \\
\hline 1081 & 5832 & 4.05 & -0.66 & 1.07 & 41 & -0.64 & 0.19 & 9 & -0.74 & 0.11 \\
\hline 975 & 5832 & 4.05 & -0.62 & 1.07 & 39 & -0.64 & 0.18 & 6 & -0.67 & 0.19 \\
\hline
\end{tabular}

comparisons, one for a pair of subgiant stars and one for two dwarfs.

Comparing the observed typical errors in EWs with the expectations of the Cayrel' formula, we see that another error source is missing, giving residual uncertainties of $2.5 \mathrm{~m} \AA$ (quadratic sum) for SGB stars and $4.1 \mathrm{~m} \AA$ for TO stars. This can be likely attributed to uncertainties in the location of the continuum (neglected in the Cayrel formula); with our procedure (relationship between FWHM and central depth), and considering for simplicity a triangular shape for the lines, we estimate that errors in the (automatic) continuum tracing at a level of $1 \%$ (for subgiants) or $2 \%$ (for dwarfs) can explain the residual discrepancy.

\subsubsection{Evaluation of errors in atmospheric parameters}

Table 3 shows the sensitivity of the derived abundances to variations in the adopted atmospheric parameters for $\mathrm{Fe}$ and other elements; this is obtained by re-iterating the analysis while varying each time only one of the parameters. This exercise was repeated for a subgiant (star 435) and a dwarf (star 1081).

Note that in some cases the entities of variations listed in Table 3 are likely to be overestimates of the actual errors in the atmospheric parameters. For instance, uncertainties in temperatures were estimated in Paper I to be $\pm 90 \mathrm{~K}$ for the dwarfs and $\pm 60 \mathrm{~K}$ for the subgiants. The same values are applicable here.

Errors in the surface gravities can be estimated by taking into account uncertainties in distance moduli and bolometric corrections (affecting luminosities: $\pm 0.06 \mathrm{dex}$ ), temperatures $( \pm 0.03 \mathrm{dex})$, and masses $( \pm 0.04 \mathrm{dex})$. If we sum in quadrature all these contributions, we find that the adopted gravities have internal errors not larger than 0.08 dex.

Typical errors in the overall metal abundance are less than 0.10 dex, and have a small effect in the final error budget.

To estimate the proper error bars in the microturbulent velocity values, we started from the original atmospheric parameters adopted for the subgiant 435 and the dwarf 1081; then we used the same set of Fe lines to repeat the analysis changing the $v_{\mathrm{t}}$ value until the $1 \sigma$ value from the slope of the abundance/line strength relation was reached. A simple comparison allows us to give an estimate of $1 \sigma$ internal errors associated to $v_{\mathrm{t}}$ : they are about $0.7 \mathrm{~km} \mathrm{~s}^{-1}$ for subgiants and $0.2 \mathrm{~km} \mathrm{~s}^{-1}$ for TO stars.

Column 7 of Table 3 allows to estimate the effect of errors in the $E W$; this was obtained by weighting the average error from a single line (obtained separately from Fe for the 9 subgiants and the 3 dwarfs) with the square root of the mean number of lines (listed in Col. 6 of the Table) measured for each element in subgiants and dwarf respectively. Finally, the last column reports the total error bars as the quadratic sum of all the contributions.

For 47 Tuc we derived from our entire sample of 12 stars an average $\mathrm{Fe}$ abundance of $[\mathrm{Fe} / \mathrm{H}]=-0.67 \pm 0.01 \pm 0.04 \mathrm{dex}$, where the first is the internal error (standard deviation of the mean) and the second represents the systematic errors, as the weighted average of errors found for TO stars and subgiants. As stated in Paper I, systematic errors in our case are mainly due to uncertainties in the temperature scale, which is related to the scale defined by field stars analyzed in the very same way of our cluster stars.

Note that the Fe solar abundances from the reference analysis made using the solar model from the Kurucz (1995) grid, with no overshooting, are slightly different from those used in Paper I, due to the different treatment of collisional damping (see Gratton et al. 2003b). The present adopted values are $\log n(\mathrm{Fe})=7.54$ for neutral iron and 7.49 for singly ionized iron.

We found no difference in the average Fe abundance between subgiant and dwarf stars in 47 Tuc: $[\mathrm{Fe} / \mathrm{H}]=-0.67 \pm$ 0.02 (9 stars) and $[\mathrm{Fe} / \mathrm{H}]=-0.67 \pm 0.01$ (3 stars), respectively. As far as Fe is concerned, 47 Tuc seems to be a very homogeneous cluster: the rms scatter from star to star is not larger than 0.05 dex, i.e. no more than $12 \%$ for this cluster. This value can be entirely explained by internal errors, suggesting a very small real star-to-star scatter in Fe abundances. This scatter well agrees with the upper limit of $\sim 0.04$ dex estimated by Hesser et al. (1987) from the photometric intrinsic width of the main sequence in 47 Tuc. 
Table 3. Sensitivities of abundance ratios to errors in the atmospheric parameters and in the equivalent widths.

\begin{tabular}{|c|c|c|c|c|c|c|c|}
\hline Ratio & $\begin{array}{c}\Delta T_{\text {eff }} \\
(+100 \mathrm{~K})\end{array}$ & $\begin{array}{c}\Delta \log g \\
(+0.2 \text { dex })\end{array}$ & $\begin{array}{c}\Delta[\mathrm{A} / \mathrm{H}] \\
(+0.2 \mathrm{dex})\end{array}$ & $\begin{array}{c}\Delta v_{\mathrm{t}} \\
\left(+0.2 \mathrm{~km} \mathrm{~s}^{-1}\right)\end{array}$ & $<N>$ & $\Delta E W$ & $\begin{array}{l}\text { tot. } \\
\text { (dex) }\end{array}$ \\
\hline & \multicolumn{7}{|c|}{ Star 435 (SGB) } \\
\hline$[\mathrm{O} / \mathrm{Na}]$ & -0.046 & -0.005 & -0.035 & +0.003 & & +0.128 & 0.132 \\
\hline$[\mathrm{O} / \mathrm{Fe}] \mathrm{I}$ & -0.084 & +0.016 & -0.071 & +0.007 & 2 & +0.099 & 0.119 \\
\hline$[\mathrm{Na} / \mathrm{Fe}] \mathrm{I}$ & -0.038 & +0.021 & -0.036 & +0.004 & 3 & +0.081 & 0.088 \\
\hline$[\mathrm{Mg} / \mathrm{Fe}] \mathrm{I}$ & -0.046 & +0.007 & -0.017 & +0.005 & 3 & +0.081 & 0.088 \\
\hline$[\mathrm{Al} / \mathrm{Fe}] \mathrm{I}$ & -0.043 & +0.020 & -0.034 & +0.006 & 5 & +0.063 & 0.074 \\
\hline$[\mathrm{Si} / \mathrm{Fe}] \mathrm{I}$ & -0.097 & +0.042 & +0.004 & +0.004 & 3 & +0.081 & 0.103 \\
\hline$[\mathrm{Ca} / \mathrm{Fe}] \mathrm{I}$ & -0.010 & -0.031 & -0.007 & +0.001 & 13 & +0.039 & 0.043 \\
\hline$[\mathrm{Sc} / \mathrm{Fe}] \mathrm{II}$ & +0.035 & -0.003 & -0.002 & -0.002 & 4 & +0.070 & 0.073 \\
\hline$[\mathrm{Ti} / \mathrm{Fe}] \mathrm{I}$ & +0.024 & -0.016 & -0.013 & -0.001 & 11 & +0.042 & 0.046 \\
\hline$[\mathrm{Ti} / \mathrm{Fe}] \mathrm{II}$ & +0.051 & -0.042 & +0.021 & -0.001 & 5 & +0.063 & 0.074 \\
\hline$[\mathrm{V} / \mathrm{Fe}] \mathrm{I}$ & +0.037 & +0.011 & -0.030 & +0.002 & 5 & +0.063 & 0.069 \\
\hline$[\mathrm{Cr} / \mathrm{Fe}] \mathrm{I}$ & -0.005 & -0.003 & -0.018 & -0.001 & 10 & +0.044 & 0.045 \\
\hline$[\mathrm{Cr} / \mathrm{Fe}] \mathrm{II}$ & -0.005 & +0.002 & -0.019 & +0.000 & 4 & +0.070 & 0.071 \\
\hline$[\mathrm{Mn} / \mathrm{Fe}] \mathrm{I}$ & -0.004 & -0.014 & -0.006 & -0.002 & 7 & +0.053 & 0.054 \\
\hline$[\mathrm{Fe} / \mathrm{H}] \mathrm{I}$ & +0.097 & -0.028 & +0.034 & -0.006 & 56 & +0.019 & 0.068 \\
\hline$[\mathrm{Fe} / \mathrm{H}] \mathrm{II}$ & -0.032 & +0.065 & +0.066 & -0.007 & 8 & +0.049 & 0.075 \\
\hline$[\mathrm{Ni} / \mathrm{Fe}] \mathrm{I}$ & -0.032 & +0.042 & -0.004 & -0.001 & 10 & +0.044 & 0.053 \\
\hline$[\mathrm{Zn} / \mathrm{Fe}] \mathrm{I}$ & -0.090 & +0.032 & +0.041 & +0.000 & 2 & +0.099 & 0.116 \\
\hline \multirow[t]{2}{*}{ Ratio } & $\begin{array}{c}\Delta T_{\text {eff }} \\
(+100 \mathrm{~K})\end{array}$ & $\begin{array}{c}\Delta \log g \\
(+0.2 \text { dex }) \\
\end{array}$ & $\begin{array}{c}\Delta[\mathrm{A} / \mathrm{H}] \\
(+0.2 \mathrm{dex}) \\
\end{array}$ & $\begin{array}{c}\Delta v_{\mathrm{t}} \\
\left(+0.2 \mathrm{~km} \mathrm{~s}^{-1}\right)\end{array}$ & $<N>$ & $\Delta E W$ & $\begin{array}{c}\text { tot. } \\
\text { (dex) }\end{array}$ \\
\hline & \multicolumn{7}{|c|}{ Star $1081(\mathrm{TO})$} \\
\hline$[\mathrm{O} / \mathrm{Na}]$ & -0.043 & -0.022 & -0.028 & +0.003 & & +0.219 & 0.224 \\
\hline$[\mathrm{O} / \mathrm{Fe}] \mathrm{I}$ & -0.075 & -0.032 & -0.026 & +0.033 & 3 & +0.110 & 0.138 \\
\hline$[\mathrm{Na} / \mathrm{Fe}] \mathrm{I}$ & -0.032 & -0.010 & +0.002 & +0.030 & 1 & +0.190 & 0.195 \\
\hline$[\mathrm{Mg} / \mathrm{Fe}] \mathrm{I}$ & -0.051 & +0.003 & -0.009 & +0.034 & 2 & +0.134 & 0.148 \\
\hline$[\mathrm{Si} / \mathrm{Fe}] \mathrm{I}$ & -0.053 & +0.013 & +0.000 & +0.028 & 1 & +0.190 & 0.199 \\
\hline$[\mathrm{Ca} / \mathrm{Fe}] \mathrm{I}$ & -0.023 & -0.002 & -0.004 & +0.015 & 11 & +0.057 & 0.064 \\
\hline$[\mathrm{Sc} / \mathrm{Fe}] \mathrm{II}$ & +0.018 & +0.003 & +0.006 & +0.026 & 3 & +0.110 & 0.114 \\
\hline$[\mathrm{Ti} / \mathrm{Fe}] \mathrm{I}$ & -0.002 & +0.011 & -0.006 & +0.001 & 5 & +0.085 & 0.085 \\
\hline$[\mathrm{Ti} / \mathrm{Fe}] \mathrm{II}$ & +0.031 & -0.023 & +0.010 & -0.021 & 8 & +0.067 & 0.078 \\
\hline$[\mathrm{V} / \mathrm{Fe}] \mathrm{I}$ & -0.083 & +0.100 & +0.023 & +0.026 & 2 & +0.134 & 0.168 \\
\hline$[\mathrm{Cr} / \mathrm{Fe}] \mathrm{I}$ & -0.013 & +0.016 & -0.007 & +0.002 & 8 & +0.067 & 0.069 \\
\hline$[\mathrm{Cr} / \mathrm{Fe}] \mathrm{II}$ & -0.010 & -0.001 & -0.012 & +0.021 & 3 & +0.110 & 0.112 \\
\hline$[\mathrm{Mn} / \mathrm{Fe}] \mathrm{I}$ & +0.000 & -0.006 & +0.006 & +0.010 & 4 & +0.095 & 0.096 \\
\hline$[\mathrm{Fe} / \mathrm{H}] \mathrm{I}$ & +0.090 & -0.022 & +0.009 & -0.044 & 39 & +0.030 & 0.105 \\
\hline$[\mathrm{Fe} / \mathrm{H}] \mathrm{II}$ & -0.004 & +0.076 & +0.030 & -0.048 & 7 & +0.072 & 0.110 \\
\hline$[\mathrm{Ni} / \mathrm{Fe}] \mathrm{I}$ & -0.022 & +0.027 & -0.005 & +0.026 & 3 & +0.110 & 0.116 \\
\hline$[\mathrm{Zn} / \mathrm{Fe}] \mathrm{I}$ & -0.050 & +0.042 & +0.014 & +0.004 & 3 & +0.110 & 0.123 \\
\hline
\end{tabular}

The new average metallicity agrees well (within the quoted uncertainties) with the value derived in Carretta \& Gratton (1997) from 5 red giants: $[\mathrm{Fe} / \mathrm{H}]=-0.70 \pm 0.03$. As a further comparison, the revised cluster abundance based on individual stellar abundances of Fe II, as given recently by Kraft \& Ivans (2003) is $[\mathrm{Fe} / \mathrm{H}]=-0.63 \mathrm{dex}$. At face value, the stars in this cluster share the same amount of iron in their photospheres, irrespective of their evolutionary status. A similar result has been obtained for other clusters as well (NGC 6397: Gratton et al. 2001; M 5: Ramirez \& Cohen 2003; M 71: Ramirez \& Cohen 2002).

\subsubsection{The ionization and excitation equilibria}

We can check the uncertainties in Fe abundances using the ionization and excitation equilibria as probes, since temperature and gravities were not derived from our spectra, but independently from colours and theoretical isochrones (see also the discussion in Carretta et al. 2002). In Fig. 4 we plot the differences between abundances obtained from neutral and singly ionized Fe lines and the slopes of the abundances from neutral lines $\log n(\mathrm{Fe} \mathrm{I})$ with respect to the excitation potential $\chi$ as a function of effective temperature.

From the upper panel of this figure we see that on average the lines of singly ionized Fe give slightly larger abundances than neutral lines (mean difference +0.08 dex, rms scatter $\sigma=0.10,12$ stars). The existence of a trend with temperature is questionable if we take into consideration the error bars. The largest differences are found among the subgiants, whereas the average difference for the 3 dwarfs is opposite in $\operatorname{sign}\left(\Delta[\mathrm{Fe} / \mathrm{H}]_{\mathrm{II}-\mathrm{I}}=-0.02, \sigma=0.03 \mathrm{dex}\right)$, and could as well be a reflection of the same difference in our solar reference values 

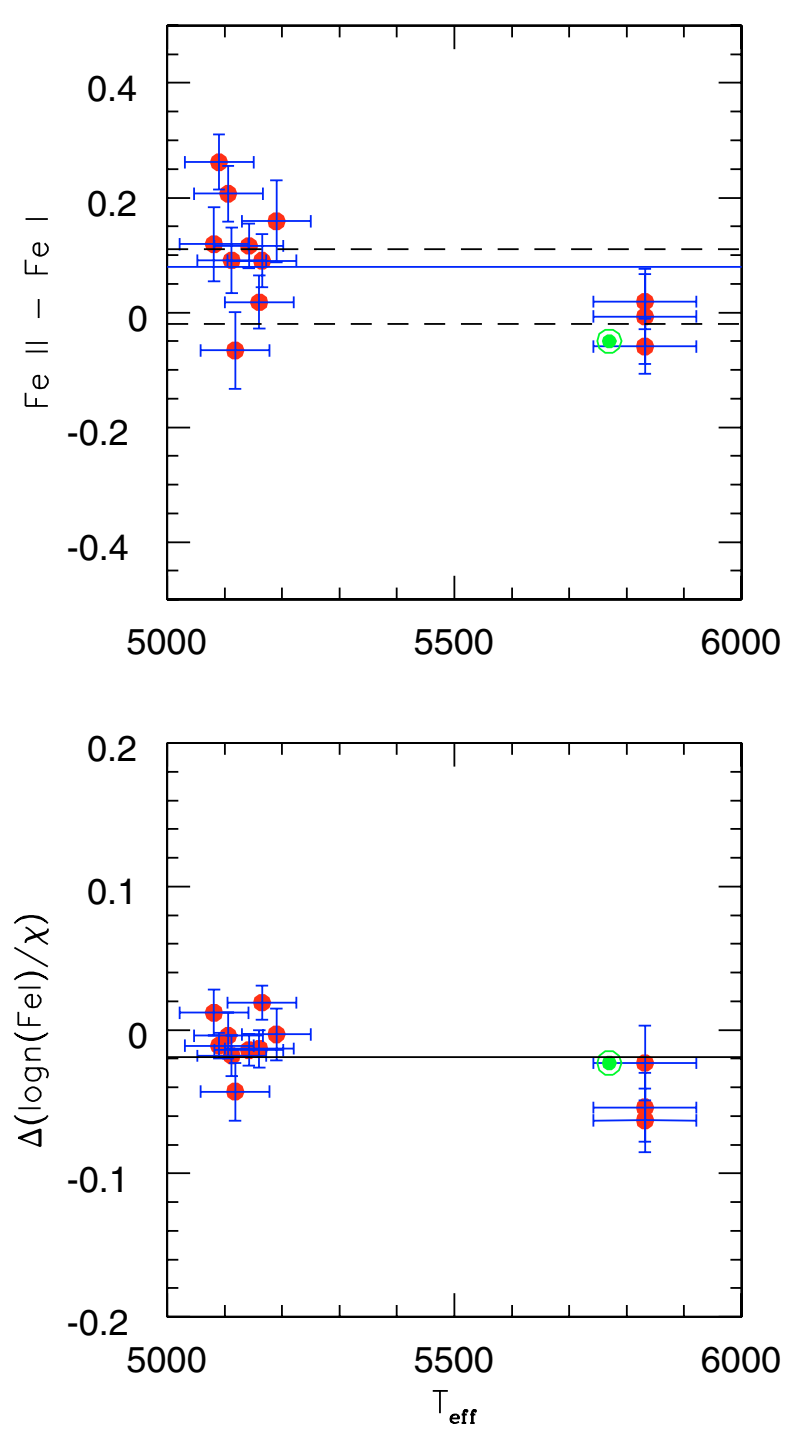

Fig. 4. Upper panel: differences between the abundances of $\mathrm{Fe}$ from singly ionized and neutral lines as a function of effective temperature for stars in 47 Tuc. The solid line is the average difference for the entire sample; the lower dashed line is the average difference for the 3 dwarfs and the upper dashed line represents the average difference for the subgiant stars. The difference for the Sun, obtained from our reference analysis, is also plotted. Lower panel: the slopes of the relationship resulting between the $\mathrm{Fe} \mathrm{I}$ abundances and the excitation potential as a function of adopted $T_{\text {eff }}$ 's. The Sun position in the plot is also marked and the solid line is the average value for our total sample.

( -0.05 dex, see above and Fig. 4, upper panel). Do we have to take into account possible departures from the LTE assumption? The dominating effect being overionization, this could explain the difference observed among subgiants; however, the good agreement found when comparing abundances for stars in different evolutionary phases does not support this hypothesis. On the other hand, errors in atmospheric parameters may affect this difference.

We started from quoted errors in temperature $( \pm 60 \mathrm{~K}$ and $\pm 90 \mathrm{~K}$ for subgiants and dwarfs respectively), and used the approximate slope of the isochrone in the subgiant/dwarf region $\Delta \log g / \Delta T_{\text {eff }}$ (about $0.05 \mathrm{dex} / 100 \mathrm{~K}$ ) to estimate the corresponding errors in gravity. We then read from Table 3 the changes in surface gravity and overall metal abundance $[\mathrm{A} / \mathrm{H}]$ induced by typical errors in temperature, computing the resulting changes in Fe I and Fe II abundance. The average observed value of $\Delta[\mathrm{Fe} / \mathrm{H}]_{\mathrm{II}-\mathrm{I}}$ of $+0.08 \mathrm{dex}$ (and more than half of the average difference for subgiants) is reproduced for temperatures that are lower by 60 (and $90 \mathrm{~K}$ ) than those presently adopted. Most of the difference therefore could simply be due to a temperature scale too low, although well within the error bars.

Further supporting evidence comes from the observed rms scatter $(0.10$ dex $)$ observed in the difference $\Delta[\mathrm{Fe} / \mathrm{H}]_{\mathrm{II}-\mathrm{I}}$. From the last Column of Table 3 , the predicted scatters are 0.06 dex and 0.08 dex for subgiant and dwarfs, respectively. Again, a significant fraction of the observed scatter is explained only by the combination of errors, both in atmospheric parameters and measured $E W \mathrm{~s}$.

Finally, the lower panel of Fig. 4 confirms that our adopted temperature scale cannot be grossly wrong, since there is no noticeable trend of derived abundances as a function of excitation potential. The average value of the slope $\Delta(\log \mathrm{Fe}) / \chi$ we derive $(-0.019 \mathrm{dex} / \mathrm{eV})$ compares well with that derived from the solar analysis $(-0.023, \sigma=0.009 \mathrm{dex} / \mathrm{eV})$ by Carretta et al. (2002), using a very similar line list.

\section{Analysis and derived abundances}

In the present work we will study only the abundances of the light elements $\mathrm{O}, \mathrm{Na}, \mathrm{Al}$, the group of the $\alpha$-elements and the Fe-groups elements. Abundances of neutron capture elements in 47 Tuc (as well as in NGC 6752 and NGC 6397) will be presented in James et al. (2003, in preparation). The carbon and nitrogen abundances, as well as the ${ }^{12} \mathrm{C} /{ }^{13} \mathrm{C}$ isotopic ratios, derived from spectral synthesis of $\mathrm{CH}$ and $\mathrm{CN}$ molecular bands for stars in 47 Tuc will be presented and discussed in a forthcoming paper (Carretta et al. 2003b, in preparation).

\subsection{Lithium}

Abundances of lithium in stars of 47 Tuc will be discussed in detail in a forthcoming paper. Here we point out that abundances of $\mathrm{Li}$ and $\mathrm{Na}$ seem to be anticorrelated among 47 Tuc dwarfs. The dwarf star with the largest equivalent width of the Li $6707 \AA$ doublet $(39.1 \mathrm{~m} \AA)$ is also the one with the lowest measured $\mathrm{Na}$ abundance $([\mathrm{Na} / \mathrm{Fe}]=-0.34 \mathrm{dex}$, star 1081 , see below). The dwarf star with the lowest measured equiva-

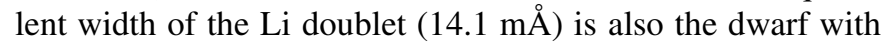
the highest $\mathrm{Na}$ abundance ( $\operatorname{star} 975,[\mathrm{Na} / \mathrm{Fe}]=+0.22$ ). In summary, our preliminary data seem to indicate that in 47 Tuc, turnoff stars with the largest $\mathrm{Na}$ abundance also show the largest Li depletion in their photospheres.

\subsection{Oxygen}

Oxygen abundances in these warm stars were derived exclusively from the permitted near-IR triplet at 7771-75 ̊. In cooler subgiants, only some of the lines were measured by our automatic procedure, while in warmer dwarfs all the lines of the triplet were successfully measured. Line measurements were further checked by eye on the spectra. Only upper limits were 
Table 4. Abundances of $\mathrm{O}, \mathrm{Na}$ and $\mathrm{Al}$ in stars of 47 Tuc.

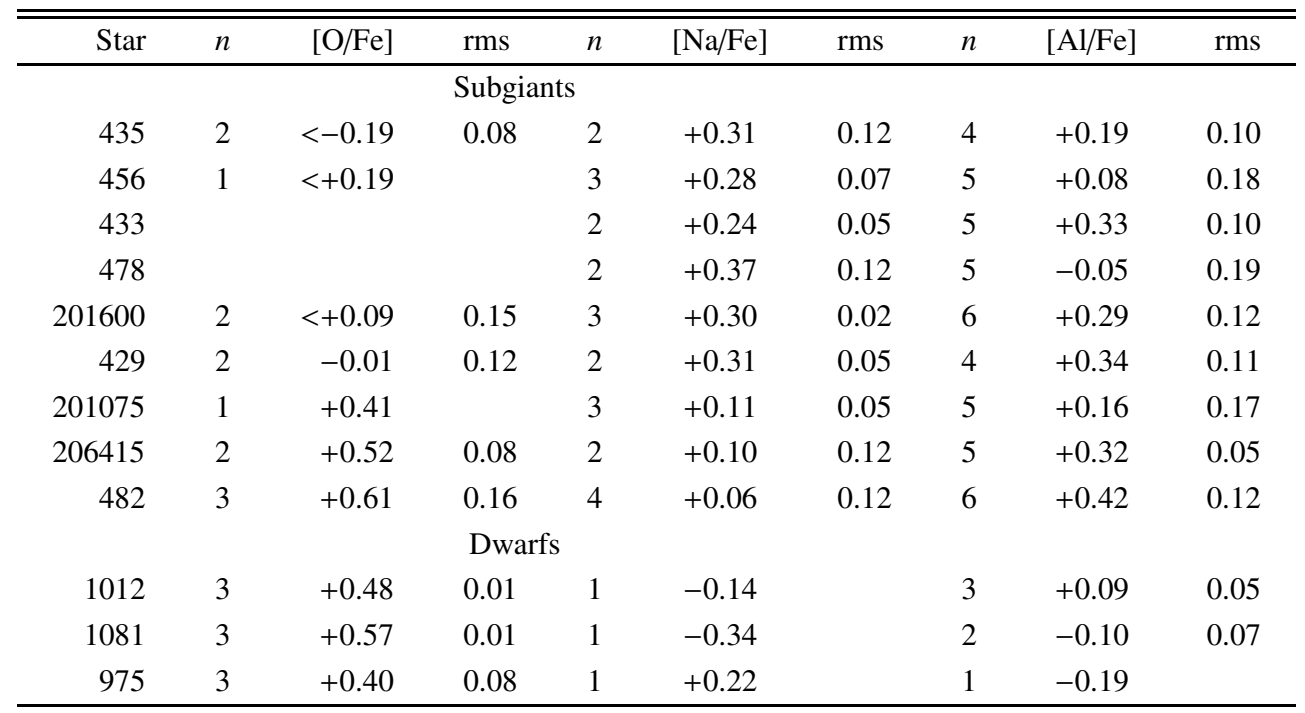

derived for three SGB stars. Final abundances and upper limits are given in Table 4, corrected for non-LTE effects as described in Gratton et al. (1999), from statistical equilibrium calculations based on empirically calibrated collisional H I cross sections. As stated in Carretta et al. (2000b), these corrections are on average rather small and their adoption brings the $\mathrm{O}$ abundances derived from the permitted triplet in good agreement with those from the forbidden [OI] line at $6300 \AA$, as far as stars with $T_{\text {eff }}>4600 \mathrm{~K}$ are considered, which is clearly our case (see also the discussion in Gratton et al. 2003b).

We carefully looked for the forbidden [OI] line at $6300 \AA$ in the spectra of all observed subgiants. Unfortunately, due to the cluster low radial velocity, in most of stars the [OI] line falls under the telluric emission line. However, in star 201075 the radial velocity shifts the emission slightly away, so that the stellar [OI] line could be measured. Using direct integration, we obtained an $E W$ of $10 \mathrm{~m} \AA$. To evaluate the $\mathrm{O}$ abundance, we must take into proper account the contribution of the Ni I line at $6300.33 \AA$ (see Allende-Prieto et al. 2001; Johanson et al. 2003). With the adopted atmospheric parameters and the derived $\mathrm{Ni}$ abundance (see below), the $\mathrm{Ni}$ line should contribute to $1 \mathrm{~m} \AA$ in this star. The resulting $E W$ of $9 \mathrm{~m} \AA$ for the [OI] line gives an oxygen abundance of $[\mathrm{O} / \mathrm{Fe}]=+0.34 \mathrm{dex}$, that compares well with that of +0.41 dex from the IR permitted triplet.

\subsection{Sodium}

47 Tuc is about 0.8 dex more metal-rich than NGC 6752, studied in Paper I. Hence, we were able to measure a number of additional $\mathrm{Na}$ I lines, beside those of the strong infrared doublet at 8183-8194 $\AA$. Lines of the doublet at 6154-6160 ̊ were observed in all subgiants and several measurements of the weak line at $4751 \AA$ were also made. The doublet at 5682-88 $\AA$ is not covered by our spectra. These lines are listed as clean in the solar analysis by Holweger (1971). Corrections for departures from LTE were taken from Gratton et al. (1999), using collisional cross sections calibrated as in Carretta et al. (2000b).
These corrections are not large (on average, $[\mathrm{Na} / \mathrm{Fe}]_{\text {non-LTE }}-$ $[\mathrm{Na} / \mathrm{Fe}]_{\mathrm{LTE}}=-0.07 \pm 0.01, \mathrm{rms}=0.05 \mathrm{dex}$ for 12 stars $)$, and they improve the agreement between abundances derived from different indicators. For example, the average difference in $\mathrm{Na}$ abundances obtained from the line at $8183 \AA$ and those derived as the mean from the doublet lines at $6154-60 \AA$ is only $-0.07 \mathrm{dex}$ ( $\mathrm{rms}=0.06,5 \mathrm{stars}$ ). Results corrected for the non-LTE effects are given in Table 4.

\subsection{Aluminum}

The strong AlI resonance lines at 3944/61 A were not measured; they are heavily saturated and likely affected by large departures from LTE (Baumüller \& Gehren 1997). Moreover, these lines are located on the wings of the strong $\mathrm{Ca}$ II $\mathrm{H}$ and $\mathrm{K}$ lines, in a region where the $S / N$ ratios of our spectra is rather low. Instead, we updated the line list of Paper I (that was limited to the doublet at 6696-98 $\AA$ ) including also the doublets at 7835-36 $\AA$ and 8772-73 $\AA$, with oscillator strengths from Lambert \& Warner (1968). Our derived Al abundances rest on this set of lines. The non-LTE effects are smaller for high excitation $\mathrm{Al}$ lines as the ones used here (see Baümuller \& Gehren 1997). Since the amount of correction for the subordinate doublets at 6696/6698 $\AA$, 7835/7836 $\AA$ and 8772/8773 $\AA$ increases toward decreasing metal abundance, we do not include any correction for the $\mathrm{Al}$ abundances derived for the metal-rich cluster 47 Tuc, even if a constant offset of about 0.15 dex cannot be excluded (see Table 2 in Baumuller \& Gehren 1997). $\mathrm{Al}$ abundances are summarized in Table 4.

\section{5. $\alpha$-elements}

A number of lines for several elements produced by $\alpha$-capture were measured. Results for $\mathrm{Mg}, \mathrm{Si}, \mathrm{Ca}$ and $\mathrm{Ti}$ are given in Table 5, together with the number of lines measured and the rms values obtained for each species. Abundances from Ti lines in two different stages of ionization allow us to check for 
Table 5. Abundance of $\alpha$-elements in stars of 47 Tuc.

\begin{tabular}{rccccccccccccccc}
\hline \hline Star & $n$ & {$[\mathrm{Mg} / \mathrm{Fe}]$} & $\mathrm{rms}$ & $n$ & {$[\mathrm{SiI} / \mathrm{Fe}]$} & $\mathrm{rms}$ & $n$ & {$[\mathrm{CaI} / \mathrm{Fe}]$} & $\mathrm{rms}$ & $n$ & {$[\mathrm{TiI} / \mathrm{Fe}]$} & $\mathrm{rms}$ & $n$ & {$[\mathrm{TiII} / \mathrm{Fe}]$} & $\mathrm{rms}$ \\
\hline & & & & & & \multicolumn{7}{c}{ Subgiants } \\
435 & 3 & +0.31 & 0.05 & 2 & +0.33 & 0.16 & 15 & +0.18 & 0.14 & 19 & +0.37 & 0.10 & 6 & +0.36 & 0.14 \\
456 & 3 & +0.32 & 0.13 & 3 & +0.30 & 0.14 & 14 & +0.24 & 0.13 & 9 & +0.31 & 0.10 & 6 & +0.47 & 0.13 \\
433 & 3 & +0.49 & 0.19 & 2 & +0.21 & 0.17 & 14 & +0.22 & 0.13 & 12 & +0.17 & 0.13 & 8 & +0.25 & 0.15 \\
478 & & & & 3 & +0.10 & 0.20 & 12 & +0.11 & 0.13 & 14 & +0.14 & 0.20 & 6 & +0.58 & 0.09 \\
201600 & 3 & +0.33 & 0.06 & 2 & +0.23 & 0.01 & 11 & +0.24 & 0.14 & 13 & +0.27 & 0.14 & 5 & +0.50 & 0.10 \\
429 & 3 & +0.39 & 0.19 & 3 & +0.42 & 0.02 & 14 & +0.09 & 0.14 & 9 & +0.17 & 0.19 & 5 & +0.28 & 0.12 \\
201075 & 3 & +0.42 & 0.09 & 4 & +0.38 & 0.15 & 13 & +0.20 & 0.14 & 10 & +0.37 & 0.10 & 4 & +0.49 & 0.04 \\
206415 & 2 & +0.34 & 0.08 & 3 & +0.45 & 0.15 & 13 & +0.27 & 0.15 & 11 & +0.24 & 0.12 & 6 & +0.31 & 0.07 \\
482 & 3 & +0.64 & 0.14 & 3 & +0.28 & 0.17 & 10 & +0.23 & 0.12 & 10 & +0.32 & 0.10 & 3 & +0.15 & 0.05 \\
& & & & & & & Dwarfs & & & & & & \\
1012 & 1 & +0.45 & & 1 & +0.03 & & 12 & +0.13 & 0.11 & 9 & +0.21 & 0.14 & 8 & +0.35 & 0.11 \\
1081 & 3 & +0.50 & 0.14 & 1 & +0.13 & & 12 & +0.14 & 0.11 & 6 & +0.22 & 0.14 & 8 & +0.49 & 0.18 \\
975 & & & & 2 & -0.10 & 0.22 & 9 & +0.21 & 0.12 & 1 & +0.06 & & & \\
\hline
\end{tabular}

Table 6. Abundances of Fe-group elements in stars of 47 Tuc: Sc to Cr.

\begin{tabular}{rlcccccccccccc}
\hline \hline Star & $n$ & {$[\mathrm{Sc} / \mathrm{Fe}] \mathrm{II}$} & $\mathrm{rms}$ & $n$ & {$[\mathrm{~V} / \mathrm{Fe}] \mathrm{I}$} & $\mathrm{rms}$ & $n$ & {$[\mathrm{Cr} / \mathrm{Fe}] \mathrm{I}$} & $\mathrm{rms}$ & $n$ & {$[\mathrm{Cr} / \mathrm{Fe}] \mathrm{II}$} & $\mathrm{rms}$ \\
\hline & & & & & & Subgiants & & & & & \\
435 & 4 & -0.03 & 0.20 & 4 & -0.02 & 0.05 & 11 & +0.18 & 0.12 & 4 & +0.00 & 0.09 \\
456 & 4 & +0.10 & 0.09 & 3 & +0.04 & 0.04 & 10 & +0.22 & 0.09 & 4 & +0.27 & 0.09 \\
433 & 4 & +0.01 & 0.11 & 5 & +0.02 & 0.13 & 6 & +0.14 & 0.12 & 4 & -0.05 & 0.12 \\
478 & 4 & +0.33 & 0.13 & 5 & +0.09 & 0.09 & 9 & +0.03 & 0.06 & 4 & +0.24 & 0.15 \\
201600 & 4 & +0.32 & 0.13 & 6 & -0.02 & 0.16 & 11 & +0.16 & 0.13 & 4 & +0.19 & 0.17 \\
429 & 3 & +0.08 & 0.14 & 7 & +0.11 & 0.12 & 8 & -0.04 & 0.11 & 4 & -0.25 & 0.17 \\
201075 & 2 & +0.04 & 0.05 & 7 & +0.08 & 0.19 & 12 & +0.11 & 0.09 & 3 & +0.24 & 0.14 \\
206415 & 3 & +0.25 & 0.10 & 7 & +0.08 & 0.13 & 9 & +0.11 & 0.13 & 5 & -0.02 & 0.12 \\
482 & 4 & +0.06 & 0.09 & 5 & +0.12 & 0.17 & 10 & +0.07 & 0.13 & 4 & -0.25 & 0.11 \\
& & & & & & Dwarfs & & & & & \\
1012 & 3 & +0.12 & 0.12 & 2 & +0.10 & 0.01 & 8 & +0.10 & 0.09 & 3 & -0.04 & 0.05 \\
1081 & 3 & +0.12 & 0.13 & & & & 8 & +0.08 & 0.13 & 4 & +0.06 & 0.07 \\
975 & 2 & -0.04 & 0.01 & & & & & & & 3 & +0.09 & 0.11 \\
\hline
\end{tabular}

additional evidence of departures from the LTE assumption. From Table 5 there is a small overabundance of singly ionized $\mathrm{Ti}$ with respect to the abundances from neutral lines (on average $[\mathrm{Ti} / \mathrm{Fe}]_{\mathrm{II}}-[\mathrm{Ti} / \mathrm{Fe}]_{\mathrm{I}}=0.12 \mathrm{dex}$, with $\mathrm{rms}=0.15 \mathrm{dex}$, 12 stars), with a hint of differences decreasing with increasing temperature among the subgiant stars. However, due to the rather limited sample size and the small range in $T_{\text {eff }}$ covered, we do not draw any further conclusions.

\subsection{Iron-group elements}

Lines of several elements belonging to the Fe-group (Sc, V, Cr, $\mathrm{Mn}, \mathrm{Ni}$ and $\mathrm{Zn}$ ) were measured. Computations of corrections due to the hyperfine structure (HFS) was performed in the relevant cases (Sc, V and $\mathrm{Mn}$ ); references are given in Gratton et al. (2003b). Another test of the adopted atmospheric parameters and of the LTE assumption is possible here since we detected lines of both neutral and singly ionized chromium.
The average difference we found is $[\mathrm{Cr} / \mathrm{Fe}]_{\mathrm{II}}-[\mathrm{Cr} / \mathrm{Fe}]_{\mathrm{I}}=$ $-0.07 \pm 0.05(\sigma=0.16)$ dex (11 stars), with no trend with temperature. Even with large scatter (mainly due to the few lines of ionized chromium measured), this finding supports our adopted scale for the atmospheric parameters.

A summary of abundances obtained for the Fe-group elements is given in Tables 6 and 7.

\section{Results and discussion}

\subsection{The Na-O anticorrelation in 47 Tuc}

Mean abundances for individual elements are summarized in Table 8 , where we used only the subgiants, to have a more homogeneous sample of stars all in the same evolutionary phase. In Col. 3, the average from available stars (Col. 2) is computed for each element ratio, and the observed star-to-star scatter is 
Table 7. Abundances of Fe-group elements in stars of 47 Tuc: Mn to Zn.

\begin{tabular}{rlllllllll}
\hline \hline Star & $n$ & {$[\mathrm{Mn} / \mathrm{Fe}] \mathrm{I}$} & $\mathrm{rms}$ & $n$ & {$[\mathrm{Ni} / \mathrm{Fe}] \mathrm{I}$} & $\mathrm{rms}$ & $n$ & {$[\mathrm{Zn} / \mathrm{Fe}] \mathrm{I}$} & $\mathrm{rms}$ \\
\hline & & & \multicolumn{7}{c}{ Subgiants } \\
435 & 8 & -0.26 & 0.07 & 7 & -0.01 & 0.11 & 2 & +0.11 & 0.18 \\
456 & 6 & -0.18 & 0.13 & 7 & +0.00 & 0.12 & 2 & +0.20 & 0.10 \\
433 & 7 & -0.40 & 0.13 & 10 & +0.04 & 0.15 & 2 & +0.15 & 0.03 \\
478 & 7 & -0.24 & 0.13 & 8 & +0.06 & 0.15 & 2 & +0.27 & 0.01 \\
201600 & 8 & -0.28 & 0.04 & 9 & +0.05 & 0.14 & 2 & +0.21 & 0.07 \\
429 & 7 & -0.39 & 0.14 & 17 & +0.09 & 0.18 & 2 & +0.11 & 0.02 \\
201075 & 6 & -0.16 & 0.13 & 11 & +0.09 & 0.12 & 3 & +0.50 & 0.09 \\
206415 & 7 & -0.37 & 0.13 & 11 & +0.20 & 0.14 & 2 & +0.03 & 0.05 \\
482 & 5 & -0.30 & 0.04 & 11 & +0.03 & 0.14 & 2 & +0.19 & 0.18 \\
& & & & Dwarfs & & & & \\
1012 & 6 & -0.33 & 0.14 & 3 & -0.25 & 0.17 & 3 & +0.05 & 0.12 \\
1081 & 3 & -0.51 & 0.10 & 3 & -0.29 & 0.13 & 3 & +0.06 & 0.18 \\
975 & 4 & -0.43 & 0.11 & 2 & +0.04 & 0.16 & & & \\
\hline
\end{tabular}

given in Col. 4. The total uncertainty, as derived from the values of Table 3, is listed in Col. 5.

Note that $\sigma_{\text {tot }}$ can be regarded as the predicted total error expected as a consequence of uncertainties in the adopted atmospheric parameters, combined with errors in $E W$ measurements. The differences give an idea of what elements show a scatter that likely exceeds the predicted observational errors in stars of 47 Tuc.

As one can see from this table, apart from cases in which the scatter is large due to the small number of lines measured (like Sc II and Cr II), two elements clearly stand out: $\mathrm{O}$ and $\mathrm{Al}^{3}$. The scatter observed in $\mathrm{Na}$ abundances would be much larger if the three turn-off stars were considered.

In panel (a) of Fig. 5 we plot the abundance ratios of oxygen and sodium from the present study. The three dwarfs and the subgiants show a well defined trend, with $[\mathrm{O} / \mathrm{Fe}]$ abundances anticorrelated with $[\mathrm{Na} / \mathrm{Fe}]$ ones. Upper limits in $\mathrm{O}$ abundances derived for three other subgiants also contribute to clearly define the anticorrelation. This is the first time that the $\mathrm{Na}-\mathrm{O}$ anticorrelation is detected among scarcely evolved stars in this cluster.

In panel (b) of this figure we added three red giants analyzed by Carretta (1994). O abundances for these stars were derived from spectral synthesis of the forbidden [OI] line at $6300 \AA$ in high resolution spectra acquired with the CASPEC spectrograph at the ESO $3.6 \mathrm{~m}$ telescope. Na abundances include the corrections for departures from LTE, using the same prescription as in Gratton et al. (1999) and in the present study.

At face value, and despite the small sample size, there seems to be no significant difference between the pattern shown by giants and less evolved stars. This impression is confirmed by panel (c) of Fig. 5, where we plot as open triangles results from a reanalysis (Carretta 1994) of 10 RGB stars observed by the Lick-Texas group (Sneden et al. 1994) in the globular

\footnotetext{
${ }^{3}$ Notice that for $\mathrm{O}$ the observed scatter is a lower limit, since it was computed including the three subgiants with upper limit to the O abundance.
}

Table 8. Mean abundance ratios for early subgiant stars in 47 Tuc and in field stars of similar metallicities.

\begin{tabular}{lrrrrrc}
\hline \hline Ratio & $N_{\text {star }}$ & Mean & $\sigma_{\text {obs }}$ & $\sigma_{\text {tot }}$ & Field & $\Delta$ \\
\hline$[\mathrm{O} / \mathrm{Fe}] \mathrm{I}$ & 7 & +0.23 & 0.27 & 0.12 & +0.53 & -0.30 \\
{$[\mathrm{Na} / \mathrm{Fe}] \mathrm{I}$} & 9 & +0.23 & 0.10 & 0.09 & +0.12 & +0.11 \\
{$[\mathrm{Mg} / \mathrm{Fe}] \mathrm{I}$} & 8 & +0.40 & 0.10 & 0.09 & +0.39 & +0.01 \\
{$[\mathrm{Al} / \mathrm{Fe}] \mathrm{I}$} & 9 & +0.23 & 0.14 & 0.07 & & \\
{$[\mathrm{Si} / \mathrm{Fe}] \mathrm{I}$} & 9 & +0.30 & 0.10 & 0.10 & +0.26 & +0.04 \\
{$[\mathrm{Ca} / \mathrm{Fe}] \mathrm{I}$} & 9 & +0.20 & 0.06 & 0.04 & +0.21 & -0.01 \\
{$[\mathrm{Sc} / \mathrm{Fe}] \mathrm{II}$} & 9 & +0.13 & 0.13 & 0.07 & +0.21 & -0.08 \\
{$[\mathrm{Ti} / \mathrm{Fe}] \mathrm{I}$} & 9 & +0.26 & 0.08 & 0.05 & +0.16 & +0.10 \\
{$[\mathrm{Ti} / \mathrm{Fe}] \mathrm{II}$} & 9 & +0.38 & 0.13 & 0.07 & +0.16 & +0.22 \\
{$[\mathrm{~V} / \mathrm{Fe}] \mathrm{I}$} & 9 & +0.05 & 0.05 & 0.07 & +0.07 & -0.02 \\
{$[\mathrm{Cr} / \mathrm{Fe}] \mathrm{I}$} & 9 & +0.11 & 0.07 & 0.05 & -0.03 & +0.14 \\
{$[\mathrm{Cr} / \mathrm{Fe}] \mathrm{II}$} & 9 & +0.04 & 0.19 & 0.07 & +0.06 & -0.02 \\
{$[\mathrm{Mn} / \mathrm{Fe}] \mathrm{I}$} & 9 & -0.29 & 0.08 & 0.05 & -0.11 & -0.18 \\
{$[\mathrm{Fe} / \mathrm{H}] \mathrm{I}$} & 9 & -0.67 & 0.05 & 0.07 & & \\
{$[\mathrm{Fe} / \mathrm{H}] \mathrm{II}$} & 9 & -0.56 & 0.09 & 0.07 & & \\
{$[\mathrm{Ni} / \mathrm{Fe}] \mathrm{I}$} & 9 & +0.06 & 0.06 & 0.05 & -0.04 & +0.10 \\
{$[\mathrm{Zn} / \mathrm{Fe}] \mathrm{I}$} & 9 & +0.20 & 0.13 & 0.12 & +0.07 & +0.13 \\
\hline
\end{tabular}

cluster M 71 (NGC 6838), a "twin" of 47 Tuc, as far as the overall metal abundance is concerned. Again, the locus involved in the $\mathrm{O}-\mathrm{Na}$ anticorrelation seems to be the same for stars in different evolutionary phases. We prefer not to use the recent results by Ramirez \& Cohen (2002) to plot only $\mathrm{Na}$ and $\mathrm{O}$ values obtained through homogeneous analyses. Their Fig. 11 is in very good agreement with the present results and lead us to conclude that, whatever the phenomenon producing the $\mathrm{Na}-$ $\mathrm{O}$ anticorrelation, it is active also in metal-rich clusters, down to scarcely evolved stars.

The extension of the $\mathrm{Na}-\mathrm{O}$ anticorrelation is not as large as that of more metal-poor cluster, but is undoubtly present. 


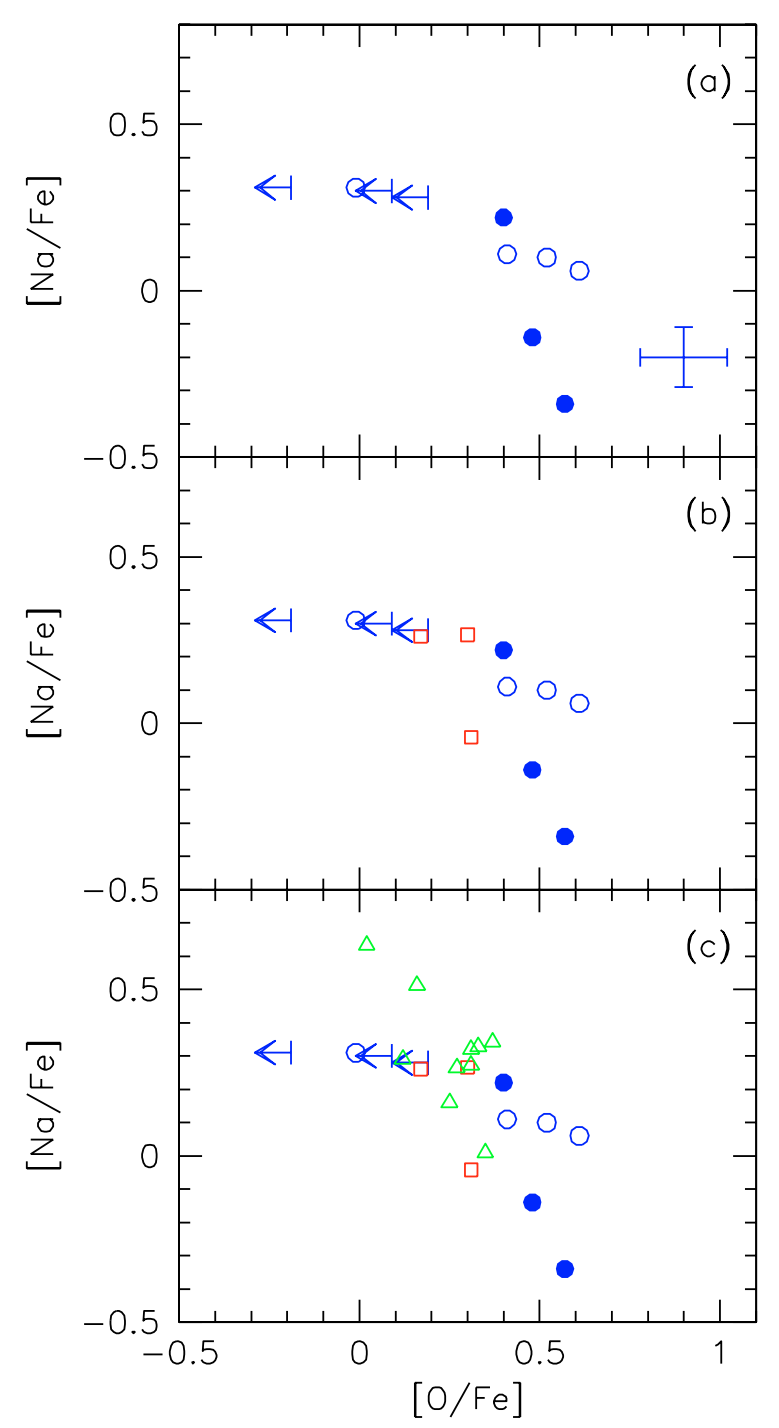

Fig. 5. $[\mathrm{Na} / \mathrm{Fe}]$ ratio as a function of $[\mathrm{O} / \mathrm{Fe}]$. Panel a): filled circles are turn-off stars and open circles are subgiant in 47 Tuc from the present study. The arrows represent the stars with only upper limits in derived $\mathrm{O}$ abundances. Panel $\mathbf{b}$ ): overimposed (open squares) to stars of this study are 3 red giant stars from Carretta (1994). Panel c): 10 red giants (open triangles) of the study of Sneden et al. (1994) in M 71 (NGC 6838), from the re-analysis by Carretta (1994), are added to the stars of the previous sources.

On the other hand, elements that are produced by p-captures at higher temperatures, such as $\mathrm{Al}$ and $\mathrm{Mg}$, do not show a clear anticorrelation, as evident in Fig. 6.

The spread in $[\mathrm{Mg} / \mathrm{Fe}]$ is not astonishing, among the subgiants, while it is somewhat larger in $[\mathrm{Al} / \mathrm{Fe}]$. However, this spread is only marginally significant (see Table 8), slightly exceeding the one expected from uncertainties due to measurement errors in the $E W \mathrm{~s}$ and to the effect of adopted atmospheric parameters ${ }^{4}$. On the other hand, $\mathrm{Al}$ is not correlated with $\mathrm{Na}$.

We note that this is not at odds with the analysis of $\mathrm{Al}$ in stars of M 71 (Ramirez \& Cohen 2002), where the existing

${ }^{4}$ The possible residual offset due to departure from LTE (see Sect. 4.4) is constant, and does not affect our conclusion on the observed spread among subgiants.

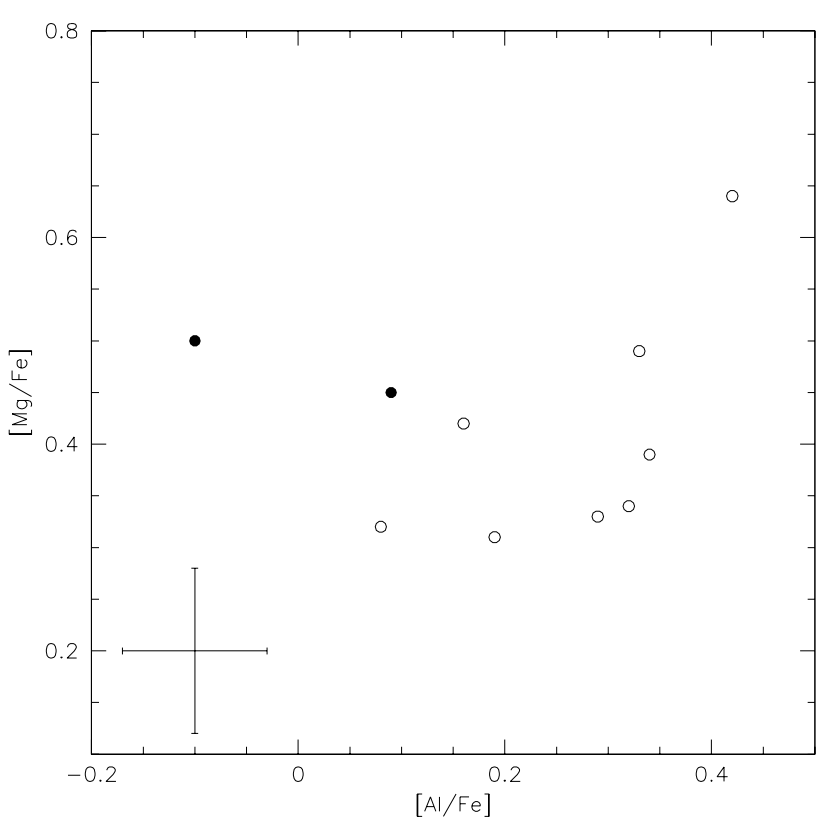

Fig. 6. Run of the $[\mathrm{Mg} / \mathrm{Fe}]$ ratio as a function of $[\mathrm{Al} / \mathrm{Fe}]$, for stars in 47 Tuc. Symbols are as in the previous figure.

spread could be explained purely as due to a combination of uncertainties in analysis (see their Table 7). This could suggest that products of p-captures in the MgAl-cycle are less likely to occur in metal-rich clusters like 47 Tuc and in M 71. Theoretical models seem to support this finding: in fact, the efficiency of the Hot Bottom Burning (HBB) in AGB stars (D'Antona 2003, private communication) is expected to decreases with increasing metal abundance and the temperature at the base of the convective envelope is likely not high enough to allow the burning of $\mathrm{Mg}$ in $\mathrm{Al}$ in the metal-rich clusters. The limited size of the observed samples precludes any further conclusion.

In summary, the pattern of light elements observed in unevolved turn-off stars and in stars at the base of the red giant branch in 47 Tuc seems to be not very different to that observed in other more metal-poor globular clusters. The range in $\mathrm{Na}$ and $\mathrm{O}$ abundances spanned by turn-off stars in 47 Tuc is almost comparable to that observed by Gratton et al. (2001) in NGC 6752; admittedly, the number of stars sampled in 47 Tuc is small.

The overall distribution of light elements shows that processes of proton-capture are at work. In the atmospheres of the studied stars we are seeing exactly the products of these reactions. In this case, the line of thought is the same as presented in Gratton et al. (2001): turn-off stars do not reach the temperature regime where the $\mathrm{ON}$ and $\mathrm{NeNa}$ cycles required to produce the $\mathrm{Na}-\mathrm{O}$ anticorrelation are active, and moreover these stars have too small convective envelopes to have efficiently mixed the ashes of these nuclear processing up to the surface. The same conclusion holds also for subgiants.

We are seeing products of nuclear burning and dredge-up in other stars, that are now not observable, but that returned their elements to the intracluster medium or directly to the surface of presently observed stars. 

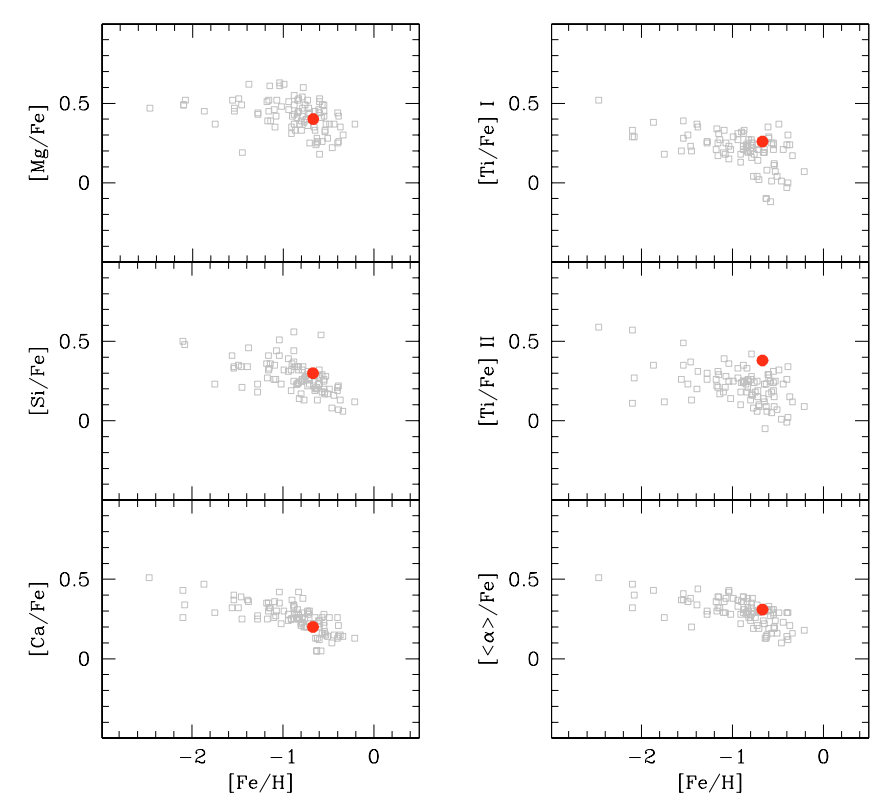

Fig. 7. Run of the overabundances of individual $\alpha$-elements as well as of the average of $\mathrm{Mg}, \mathrm{Si}, \mathrm{Ca}$ and $\mathrm{Ti}$ as a function of $[\mathrm{Fe} / \mathrm{H}]$. Open squares are the field stars belonging to the dissipative component of the Galaxy studied by Gratton et al. (2003b). The big filled circle represents present data for 47 Tuc. Note the position of the cluster at the upper envelope of the field star distribution for $\mathrm{Ti}$ and the average of $\alpha$-elements. Typical error bars for the cluster average abundances are smaller than the symbol size.

\subsection{Self-enrichment in globular clusters?}

Aside from changes in the pattern of light elements, we might expect that cluster stars could significantly differ from field stars of the same metallicity, if the protocluster was able to retain the yields of sustained and independent chemical evolution. Having obtained an extensive set of abundances of $\alpha$-elements in star of 47 Tuc, we may test this hypothesis by looking at the pattern of elements possibly enriched by the ejecta of core-collapse supernovae (Cayrel 1986; Brown et al. 1991, 1995; Parmentier et al. 1999; Parmentier \& Gilmore 2001). This suggestion is inspired by the fact that in our own Galaxy the metallicity distribution of GCs is apparently quite different from that of field stars (e.g., Carney 1993). Observationally, we might then expect that GCs have a larger $[\alpha / \mathrm{Fe}]$ ratio than field stars of similar metal abundance. This signature is likely subtle, and may have escaped detection in the study by Carney (1996); it should be more evident among the metal-rich globular clusters, because observations of field stars indicate that at metallicity $[\mathrm{Fe} / \mathrm{H}] \sim-1$ there is already a significant contribution by type Ia $\mathrm{SNe}$, which has lowered the $[\alpha / \mathrm{Fe}]$ ratio from $\sim 0.4$ to $\sim 0.3$ (see Fig. 7 ).

The elements to be considered here are those not involved in the $\mathrm{O}-\mathrm{Na}$ anticorrelation $(\mathrm{Mg}, \mathrm{Si}, \mathrm{Ti}$, and $\mathrm{Ca})$, for which very accurate abundances have been obtained. Results for 47 Tuc are compared in Fig. 7 with the overabundances measured in field stars of similar metallicity in the dissipative component of our Galaxy (Gratton et al. 2003b). Values are reported in Col. 6 of Table 8, while in Col. 8 the differences (47 Tuc minus field)

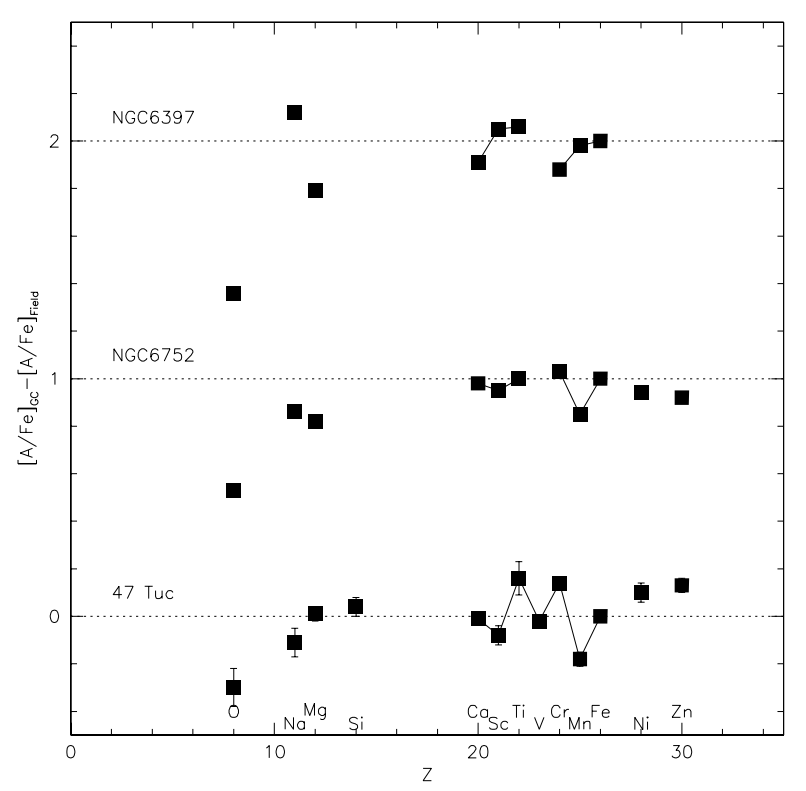

Fig. 8. Differences for several elements between the clusters analyzed in Paper I and in the present work, and field stars of similar metallicities (from Gratton et al. 2003b). Differences were arbitrarily shifted for display purposes. Solid lines connect Fe-group elements having consecutive atomic number. Notice the enhanced odd-even effect, in particular in 47 Tuc: Sc, V and Mn are clearly deficient with respect to $\mathrm{Ti}, \mathrm{Cr}$ and $\mathrm{Fe}$.

between the abundances are listed. The analysis is completely consistent with the present one.

The result for $\mathrm{Mg}$ is less significant, since, on average, $\mathrm{Mg}$ might be somewhat reduced due to its participation in the $\mathrm{Mg}-\mathrm{Al}$ anticorrelation phenomenon, although there is no evidence that this is the case for 47 Tuc. Offsets for individual elements are +0.04 dex for $\mathrm{Si},-0.01$ dex for $\mathrm{Ca}$ and $0.16 \mathrm{dex}$ for $\mathrm{Ti}$ (average of $\mathrm{Ti}$ I and $\mathrm{Ti}$ II). On average, 47 Tuc seems to be overabundant in $\alpha$-elements by about $0.06 \pm 0.04$ dex with respect to halo/thick disk stars of similar metallicities. This result is not significant.

On the other hand, this figure seems to indicate that some excess of Ti may indeed be present (a similar indication being obtained for M4 by Ivans et al. 1999), pointing out possibly that GCs produced a significant fraction of their metals. More extended observations are needed to firmly establish this point.

\subsection{Mn and the enhanced odd-even effect}

A much more puzzling result concerns the abundance of odd Fe-group elements. More than thirty years ago, Helfer et al. (1959) noted that among metal-poor stars an enhanced oddeven effect for Fe-group elements existed.

A graphical representation of the odd-even effect in globular clusters is given in Fig. 8, where the differences of abundances with respect to field stars of similar metallicities are displayed for the three clusters analyzed so far in the Large Program 165.L-0263. In GCs, the odd-number Fe-group elements show abundances that are more deficient with respect to the even-number Fe-group elements than in field stars. 
This odd-even effect seems to increase with increasing overall metallicity, reaching as much as about 0.2 dex in 47 Tuc. This exceeds the observational errors.

Here we concentrate on manganese, as the run of this element has been studied for long time in several stars; moreover, it is the one showing the most marked underabundance. On the other hand, the major shortcoming is that the nucleosynthetic site of production of $\mathrm{Mn}$ is not yet well known, although $\mathrm{Mn}$ is believed to be formed mostly in type Ia $\mathrm{SNe}$ (e.g. Nakamura et al. 1999).

Gratton (1989) pointed out that the run of the ratio $[\mathrm{Mn} / \mathrm{Fe}]$ in metal-poor stars is mirroring in some way the run of $\alpha$-elements, being almost flat at very low metallicities, then rising with increasing $[\mathrm{Fe} / \mathrm{H}]$, starting at about $[\mathrm{Fe} / \mathrm{H}]=-1$. The high, constant $[\alpha / \mathrm{Fe}]$ ratio in metal-poor stars is commonly interpreted as due to the delay with which elements are injected in the interstellar medium (ISM) as a consequence of different typical lifetimes of the producers. Massive stars ending their lifes as SN II enrich in very short times the ISM with $\alpha$-enriched yields; when the longer-lived precursors of SN Ia undergo thermonuclear explosion the major source of $\mathrm{Fe}$ is activated, thus decreasing the ratio $[\alpha / \mathrm{Fe}]$. In this framework, Gratton (1989) suggested that the observed run of $[\mathrm{Mn} / \mathrm{Fe}]$ vs. $[\mathrm{Fe} / \mathrm{H}]$ could indicate that the fraction of manganese produced by SN II is lower than the fraction that SN Ia are allowed to inject in the ISM.

However, the production of elements with odd atomic numbers in the Fe-group depends on the available neutron excess. Arnett (1971) proposed that the yields of Mn produced in SN II are then a function of the metallicity, through the neutron excess. In this view, the rise at higher metallicity would be due to the occurrence of more metal-rich supernovae.

This approach was recently revived by McWilliam et al. (2003; hereinafter McW03). They used new observations of giants in the galactic bulge and in the dwarf spheroidal Sagittarius to constrain the run of $[\mathrm{Mn} / \mathrm{Fe}]$ at low metal abundance and at $[\mathrm{Fe} / \mathrm{H}]$ values typical of the solar neighborhood. Using these two environments to pinpoint the underabundances of Mn, they claimed that manganese yields from both SN Ia and SN II are metallicity dependent.

However, since their results are based on the still few reliable high resolution spectra obtainable for these rather distant systems, we gathered $\mathrm{Mn}$ abundances for several kinds of environments, to better clarify the observational framework. Results are displayed in Fig. 9.

To have a reference, we included field stars from both the accretion and dissipative components as found by Gratton et al. (2003b), covering the range of metallicity up to $[\mathrm{Fe} / \mathrm{H}] \lessgtr-0.5$. These data were complemented at higher metallicity with thin disk stars from the study of Reddy et al. (2003), after an offset of +0.12 dex was added to their $[\mathrm{Mn} / \mathrm{Fe}]$ ratios to take into account differences in the adopted solar reference abundances. Also, we plotted data from Feltzing \& Gustaffson (1998), after subtracting 0.04 dex to mantain the offset of 0.16 dex with Reddy et al. data, as adopted in McW03.

Data points for red giants in Sgr were taken from Bonifacio et al. (2000) and were read from figures in the McW03 paper, like those for the galactic bulge.

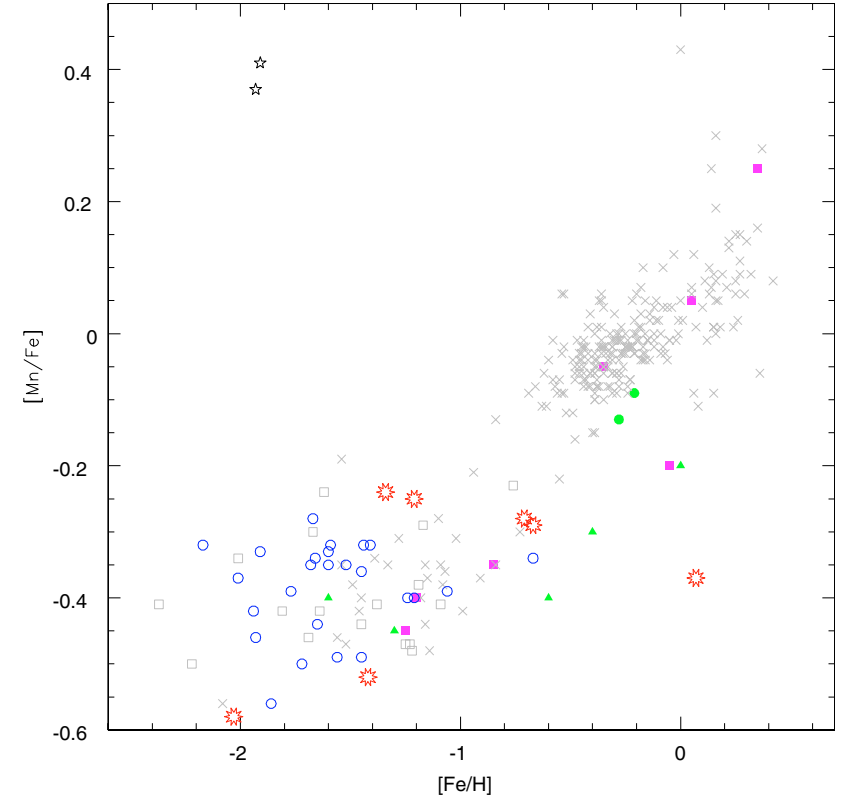

Fig. 9. Run of the $[\mathrm{Mn} / \mathrm{Fe}]$ ratio as a function of $[\mathrm{Fe} / \mathrm{H}]$ for stars in different environments. Light crosses $([\mathrm{Fe} / \mathrm{H}] \lessgtr 0.5 \mathrm{dex})$ : field stars of the dissipative component (halo/thick disk) from Gratton et al. (2003b). Light crosses $(-0.6 \lesssim[\mathrm{Fe} / \mathrm{H}] \lessgtr 0)$ : field thin disk stars from Feltzing \& Gustaffson (1998; FG98). Light crosses ([Fe/H] $\gtrsim 0)$ : thin disk stars from Reddy et al. (2000). Open squares: field stars of the accretion component, from Gratton et al. (2003b). Open circles: red giants in several dSphs from Shetrone et al. (2001, 2003). Open fivepointed stars: two low $\alpha$-elements stars from Ivans et al. (2003). Filled squares: bulge red giants, from McW03. Filled triangles: giants in the Sgr dwarf galaxy, from McW03. Filled circles: giants in Sgr from Bonifacio et al. (2000). Open "nova explosions": average abundances for galactic globular clusters: NGC 6397 and NGC 6752 (Gratton et al. 2001, $[\mathrm{Fe} / \mathrm{H}]=-2.03$ and -1.42 respectively), Pal 5 (Smith et al. 2002, $[\mathrm{Fe} / \mathrm{H}]=-1.34)$, M 5 (Ivans et al. 2001, $[\mathrm{Fe} / \mathrm{H}]=-1.24)$, M 71 (Ramirez \& Cohen 2002, $[\mathrm{Fe} / \mathrm{H}]=-0.71$ ), 47 Tuc (this work, $[\mathrm{Fe} / \mathrm{H}]=-0.67)$ and NGC $6528($ Carretta et al. 2001, $[\mathrm{Fe} / \mathrm{H}]=+0.07)$.

Several features in this diagram are worth of discussion.

1) Field stars show the same pattern noted by Gratton (1989): decreasing $[\mathrm{Mn} / \mathrm{Fe}]$ for decreasing metallicity, from super-solar values down to about $[\mathrm{Fe} / \mathrm{H}] \sim-1.6$, where a plateau, although with large scatter, can be envisioned around $[\mathrm{Mn} / \mathrm{Fe}] \sim-0.4$. Both the quoted interpretations of SN Ia producing a higher fraction of Mn with respect to SN II and/or more metal-rich SN Ia yielding more Mn than metal-poor SN Ia can explain this result.

2) Most of the red giants surveyed to date in dwarf spheroidal galaxies (dSphs, open circles in Fig. 9, from Shetrone et al. 2001, 2003) span a range in metallicity restricted between $-2<[\mathrm{Fe} / \mathrm{H}]<-1$, and the overwhelming majority is scattered around a mean value of $[\mathrm{Mn} / \mathrm{Fe}] \sim-0.4$, with no trend increasing the observed $[\mathrm{Mn} / \mathrm{Fe}]$ ratios over 1 full dex in $[\mathrm{Fe} / \mathrm{H}]$. Stars in present day dSphs seem to behave differently from those in the cannibalized dwarf Sgr (see below). Our choice of the field reference sample does not affect our conclusions; it would hold also using the sample assembled by McW03. 
3) The locus occupied by stars in dSphs seems to be coincident with that populated by the accretion component selected by Gratton et al. (2003b), in the $[\mathrm{Mn} / \mathrm{Fe}]-[\mathrm{Fe} / \mathrm{H}]$ diagram. This field population is composed of non-rotating or even counterrotating halo stars, with distinctive chemistry with respect to the dissipative component of the halo/thick disk.

Hence, as far as Mn is concerned, it is tantalizing to conclude that these stars, likely relics of objects accreted or captured in the past by our Galaxy, share the same kind of chemical signature as present-day dSphs, i.e. stellar systems with star formation occurring at a uniformly low rate, even if at different times (Tolstoy et al. 2003).

On the other hand, it is well known that the pattern of $\alpha$-elements differs between stars in dSphs and in the galactic field. The low (even subsolar) ratios observed in giant stars of present-day dwarfs suggest that the action of very massive stars was not strong enough to affect the chemical evolution in these objects (Tolstoy et al. 2003). The stars of the galactic accretion component (Gratton et al. 2003b), however, show higher $[\alpha / \mathrm{Fe}]$ ratios; it is possible that in the primordial satellites that were likely accreted in the Galaxy, leaving behind this accreted component, stars with initial masses $>15-20 M_{\odot}$ were able to contribute a higher content of $\alpha$-elements.

4) The disrupting dwarf spheroidal Sgr seems to represent a different case. The five stars analyzed by McW03 and the two by Bonifacio et al. (2000) span a much more extended range in metallicity and reach a higher overall metallicity, maybe reflecting a higher mass of the host galaxy. Sgr was probably able to retain a higher amount of gas, reaching up to solar values of $[\mathrm{Fe} / \mathrm{H}]$. The most metal poor component of Sgr seems to be intermingled with field stars, and as well with the most metal-poor component of the bulge population.

Plotting in the same panel both bulge and Sgr stars analyzed by McW03, it seems that in the high metallicity regime these populations seem to differ from the galactic field population. However considering also the two stars analyzed by Bonifacio et al. (2000) this distinction seems less clear; Mn abundances for the larger sample presented by Bonifacio et al. (2003) will allow a clearer view of the situation. The conclusion of McW03, that type Ia SNe produce metallicity-dependent yields, as far as $\mathrm{Mn}$ is concerned, is considerably weakened if the stars of Bonifacio et al. (2000) are also considered.

5) Concerning the bulge population, the stars analyzed in McW03 seem to bracket the field population. Using the new data, however, and by plotting side-by-side bulge and Sgr stars, it is quite difficult to distinguish the different populations, at the metal poor end. In our opinion, in the high metallicity regime $([\mathrm{Fe} / \mathrm{H}] \geq-0.5)$, we can only conclude that data encompass a large range of $[\mathrm{Mn} / \mathrm{Fe}]$ ratios. This impression is strengthened by the average abundance of the bulge cluster NGC 6528 (Carretta et al. 2001), which is based on four cluster stars. We therefore caution that it may be dangerous to argue about implications from $\mathrm{Mn}$ in metal-rich populations, since the scatter at solar metallicity is as large as $0.6 \mathrm{dex}$ in $[\mathrm{Mn} / \mathrm{Fe}]$.

6) The run of globular clusters in the $[\mathrm{Mn} / \mathrm{Fe}]$ vs. $[\mathrm{Fe} / \mathrm{H}]$ plane seems to be more or less flat, with some scatter. NGC 6528, as already noted, stands out, since its $[\mathrm{Mn} / \mathrm{Fe}]$ ratio is about 0.4 dex below the mean value of stars in the solar neighborhood. This cluster is also rather peculiar for its kinematics: its high radial velocity rules out the possibility that NGC 6528 is a disk cluster, but we cannot exclude that we are just seeing an inner halo cluster presently passing through the bulge (see discussion in Carretta et al. 2001). 47 Tuc, on the other hand, seems to lie under the mean locus defined by the field stars at $[\mathrm{Fe} / \mathrm{H}] \sim-0.7$.

7) Finally, a recent paper by Ivans et al. (2003) shows that the situation is much more complex. They studied in detail three well known $\alpha$-poor stars (five-pointed stars in Fig. 9; we dropped in this figure star BD+24 1676 , whose $\mathrm{Mn}$ abundance is based on only 1 line). While these stars have low amounts of $\alpha$-elements also shown by stars in dSphs, their Mn content stands out about 1 dex above the mean level of stars of similar (very low) metallicity. Moreover, it is likely that these peculiar stars formed in sub-systems that were distinct from the global galactic population and subsequently accreted, as hinted by their orbital characteristics (see Ivans et al. 2003). The interplay between the abundances (yields) of $\alpha$-elements and those of $\mathrm{Mn}$ is still poorly understood. The position of these stars argues that the yield of $\mathrm{Mn}$ and $\mathrm{Fe}$ are quite different in SN II and SN Ia, in agreement with theoretical predictions (e.g. Tsujimoto et al. 1995).

8) The situation for Mn is somehow mirrored by that of $\mathrm{Ni}$, an even element: while in the Galactic field stars Ni tracks iron very closely $([\mathrm{Ni} / \mathrm{Fe}] \sim 0.0)$ down to extremely low metallicities $([\mathrm{Fe} / \mathrm{H}] \sim-4.0$, Cayrel et al. 2004), the GCs Ruprecht 106, Pal 12 (Brown et al. 1997) and Terzan 7 (Sbordone et al. 2004) as well as the Sgr field stars (Bonifacio et al. 2002) show $[\mathrm{Ni} / \mathrm{Fe}]<0$. Pal 12 and Terzan 7 are associated with Sgr. In all these objects $[\alpha / \mathrm{Fe}]$ is solar or sub-solar. 47 Tuc behaves for Ni like the field stars and contrasts quite strikingly with Terzan 7, which has a similar metallicity. Any theory which seeks to explain anomalous $\mathrm{Mn}$ abundances should at the same time be able to explain the $\mathrm{Ni}$ abundances.

In this framework, it seems rather difficult to use our derived abundances for 47 Tuc to put strong constraints on the production of $\mathrm{Mn}$. The commonly adopted hypothesis are: (i) the contribution from SN Ia is larger than that from SN II; in this case, our results imply that type Ia $\mathrm{SNe}$ contributed less to the building up of elements in 47 Tuc with respect to field stars of similar metallicity; and (ii) Mn yields are metallicitydependent (via the neutron excess) and the SNe polluting the gas that formed 47 Tuc were more metal-poor (by about $1 \mathrm{dex}$ ) than those enriching the field stars.

In both cases, however, we might interpret these data as hints of a peculiar chemical history for 47 Tuc. Two tentative scenarios can be envisioned:

(a) the proto-cluster cloud that formed 47 Tuc was able to build up a significant fraction of its own metals;

(b) 47 Tuc might come from a larger object having a chemical evolution quite distinct from that of our Galaxy. In the scenario of an accretion of a rather large satellite in the past of our Galaxy (see e.g. Gratton et al. 2000a; Freeman \& Bland-Hawthorne 2002), "inflating" the primordial disk to produce the present thick disk, 47 Tuc could have formed out of the material of the infalling satellite during the accretion phase. To support this, we note that the age of this cluster is 
about 2 Gyr less than the mean age of typical old galactic clusters (Rosenberg et al. 1999; Gratton et al. 2003a). Moreover, among the metal-rich $([\mathrm{Fe} / \mathrm{H}]>-0.8)$ and more massive clusters, 47 Tuc is the object that lies furthest from the galactic plane $(-3 \mathrm{kpc}$, from the February 2003 update of Harris 1996). Besides, there are very few stars of similar metallicity in the dissipative component whose maximum height above the galactic plane outrank the distance at which 47 Tuc is presently observed below this plane (Gratton et al. 2003b).

We caution that the observational framework for the Mn abundances is still quite complex and poorly understood. Is it possible that 47 Tuc, one of the most massive globular clusters in the Galaxy, has been formed in a formerly independent subsystem? While at present it is not possible to give a clearcut answer with the available data, we suggest that some hints do exist from both its chemical composition and orbital features.

\section{Summary}

Using the high resolution UVES spectra we firmly established the metallicity of 47 Tuc to be $[\mathrm{Fe} / \mathrm{H}]=-0.67 \pm 0.05$, in substantial agreement with previous results in the literature. As for the more metal-poor clusters NGC 6397 and NGC 6752, we find that TO and SGB stars share the same iron abundance and there is no star-to-star scatter in excess of that expected from observational errors.

As in NGC 6752, we have been able to demonstrate the existence of a $\mathrm{Na}-\mathrm{O}$ anticorrelation which must be the signature of a previous generation of stars, since TO and SGB stars do not have high enough internal temperatures to produce such patterns, no matter how deep the mixing.

Some scatter has been found also for $\mathrm{Li}, \mathrm{Mg}$ and $\mathrm{Al}$; no clear correlations have been found for the last two elements.

For most of the other elements, 47 Tuc seems to behave as field stars of similar metallicity, with two possible exceptions: Ti seems to be marginally higher than in field stars, Mn seems to be marginally lower. The errors in our analysis and the scatter among the field stars are such that none of these anomalies is highly significant; however, if real, they could point towards the origin of 47 Tuc as part of an independent, possibly larger, system later captured by the Galaxy.

Acknowledgements. This research has made use of the SIMBAD data base, operated at CDS, Strasbourg, France, and was funded by COFIN2001028897 by Ministero Università e Ricerca Scientifica, Italy. We wish to thank F. Grundahl for providing photometry of stars in 47 Tuc. We thank the ESO staff at Paranal (Chile) for their help during observing runs and Elena Pancino for reading the manuscripts and making useful comments.

\section{References}

Alonso, A., Arribas, S., \& Martinez-Roger, C. 1996, A\&A, 313, 873 Allende Prieto, C., Lambert, D. L., \& Asplund, M. 2001, ApJ, 556, L63

Arnett, W. D. 1971, ApJ, 166, 153

Baumüller, D., \& Gehren, T. 1997, A\&A, 325, 1088

Bell, R. A., Hesser, J. E., \& Cannon, R. D. 1983, ApJ, 269, 580
Bonifacio, P., Hill, V., Molaro, P., et al. 2000, A\&A, 359, 663

Bonifacio, P., Sbordone, L., \& Marconi, G. 2003, Mem. S. A. It. Suppl., in press

Bragaglia, A., Carretta, E., Gratton, R. G., et al. 2001, AJ, 121, 327

Briley, M. M., Hesser, J. E., \& Bell, R. A. 1991, ApJ, 373, 482

Briley, M. M., Smith, V. V., Suntzeff, N. B., et al. 1996, Nature, 383, 604

Brown, J. H., Burkert, A., \& Truran, J. W. 1991, ApJ, 376, 115

Brown, J. H., Burkert, A., \& Truran, J. W. 1995, ApJ, 440, 666

Brown, J. A., Wallerstein, G., \& Zucker, D. 1997, AJ, 114, 180

Brown, J. A., \& Wallerstein, G. 1992, AJ, 104, 1818

Cannon, R. D., Croke, B. F. W., Bell, R. A., Hesser, J. E., \& Stathakis, R. A. 1998, MNRAS, 298, 601

Carney, B. W. 1993, in The Globular Clusters-Galaxy Connection, ed. G. H. Smith, \& J. P. Brodie, ASP Conf. Ser., 48, 234

Carney, B. W. 1996, PASP, 108, 900

Carretta, E. 1994, Ph.D. Thesis, University of Padova

Carretta, E., \& Gratton, R. G. 1997, A\&AS, 121, 95

Carretta, E., Gratton, R. G., Clementini, G., \& Fusi Pecci, F. 2000a, ApJ, 533, 215

Carretta, E., Gratton, R.G., \& Sneden, C. 2000b, A\&A, 356, 238

Carretta, E., Cohen, J. G., Gratton, R. G., \& Behr, B. B. 2001, AJ, 122, 1469

Carretta, E., Gratton, R. G., Cohen, J. G., Beers, T. C., \& Christlieb, N. 2002, AJ, 124, 481

Cayrel, R. 1986, A\&A, 168, 81

Cayrel, R. 1989 in The Impact of Very High S/N Spectroscopy on Stellar Physics, ed. G. Cayrel de Strobel, \& M. Spite (Dordrecht: Kluwer), 345

Cayrel, R., Depagne, E., Spite, M., et al. 2004, A\&A, 416, 1117

Cottrell, P. L., \& Da Costa, G. S. 1981, ApJ, 245, L79

Denisenkov, P. A., \& Denisenkova, S. N. 1990, Sov. Astron. Lett., 16, 275

Feltzing, S., \& Gustafsson, B. 1998, A\&AS, 129, 237

Freeman, K., \& Bland-Hawthorn, J. 2002, ARA\&A, 40, 487

Gratton, R. G. 1989, A\&A, 208, 171

Gratton, R. G., Carretta, E., Eriksson, K., \& Gustafsson, B. 1999, A\&A, 350, 955

Gratton, R. G., Carretta, E., Matteucci, F., \& Sneden, C. 2000a, A\&A, 358,671

Gratton, R. G., Sneden, C., Carretta, E., \& Bragaglia, A. 2000b, A\&A, 354, 169

Gratton, R. G., Bonifacio, P., Bragaglia, A., et al. 2001, A\&A, 369, 87

Gratton, R. G., Bragaglia, A., Carretta, E., et al. 2003a, A\&A, 408, 529

Gratton, R. G., Carretta, E., Claudi, R., Lucatello, S., \& Barbieri, M. 2003b, A\&A, 404, 187

Grundahl, F., Vandenberg, D. A., Stetson, P. B., Anderson, M. I., \& Briley, M. 1999, in The Galactic Halo: from Globular Clusters to Field Stars [astro-ph/9909447]

Harris, W. E. 1996, AJ, 112, 1487

Helfer, H. L., Wallerstein, G., \& Greenstein, J. L. 1959, ApJ, 129, 700

Hesser, J. E. 1978, ApJ, 223, L117

Hesser, J. E., \& Bell, R. A. 1980, ApJ, 238, L149

Hesser, J. E., Harris, W. E., Vandenberg, D. A., et al. 1987, PASP, 99, 739

Holweger, H. 1971, A\&A, 10, 128

Ivans, I. I., Sneden, C., James, C. R., et al. 2003, ApJ, 592, 906

Ivans, I. I., Kraft, R. P., Sneden, C., et al. 2001, AJ, 122, 1438

Johansson, S., Litzén, U., Lundberg, H., \& Zhang, Z. 2003, ApJ, 584, L107

Kraft, R. P. 1994, PASP, 106, 553

Kraft, R. P., \& Ivans, I. I. 2003, PASP, 115, 143 
Kurucz, R. L. 1995, CD-ROM 13, Smithsonian Astrophysical Observatory, Cambridge

Lambert, D. L., \& Warner, B. 1968, MNRAS, 138, 181

Langer, G. E., Hoffman, R., \& Sneden, C. 1993, PASP, 105, 301

Lucatello, S., \& Gratton, R. G. 2003, A\&A, 406, 691

McWilliam, A., Rich, M. R., \& Smecker-Hane, T. A. 2003, ApJ, 592, L21

Nakamura, T., Umeda, H., Nomoto, K., Thieleman, F.-K., \& Burrows, A. 1999, ApJ, 517, 193

Norris, J. E. 1987, ApJ, 313, 65

Norris, J. E., \& Da Costa, G. S. 1995, ApJ, 441, L81

Parmentier, G., Jehin, J., Magain, P., et al. 1999, A\&A, 352, 138

Parmentier, G., \& Gilmore, G. 2001, A\&A, 378, 97

Ramirez, S., \& Cohen, J. G. 2002, AJ, 123, 3277

Ramirez, S., \& Cohen, J. G. 2003, AJ, 125, 224

Reddy, B. E., Tomkin, J., Lambert, D. L., \& Allende Prieto, C. 2003, MNRAS, 340, 304
Rosenberg, A., Saviane, I., Piotto, G., \& Aparicio, A. 1999, AJ, 118, 2306

Sbordone, L., Bonifacio, P., Marconi, G., \& Buonanno, R. 2004, Mem. SAIt, in press

Shetrone, M. D., Côté, P., \& Sargent, W. L. W. 2001, ApJ, 548, 592

Shetrone, M. D., Venn, K. A., Tolstoy, E., et al. 2003, ApJ, 125, 684

Smith, G. H. 1987, PASP, 99, 67

Smith, G. H., \& Mateo, M. 1990, ApJ, 353, 533

Smith, G. H., Sneden, C., \& Kraft, R. P. 2002, AJ, 123, 1502

Sneden, C., Kraft, R. P., Langer, G. E., Prosser, C. F., \& Shetrone, M. D. 1994, AJ, 107, 1173

Suntzeff, N. B. 1993, ASP Conf. Ser., 48, 167

Sweigart, A. V., \& Mengel, J. G. 1979, ApJ, 229, 624

Tassoul, M., \& Tassoul, J.-L. 1984, ApJ, 279, 384

Tolstoy, E., Venn, K. A., Shetrone, M. D., et al. 2003, AJ, 125, 707

Tsujimoto, T., Nomoto, K., Yoshii, Y., et al. 1995, MNRAS, 277, 945 